\title{
Pseudo-Nambu-Goldstone dark matter and two-Higgs-doublet models
}

\author{
Xue-Min Jiang, ${ }^{1,2}$ Chengfeng Cai, ${ }^{1}$ Zhao-Huan Yu $\odot,{ }^{1, *}$ Yu-Pan Zeng $\odot,{ }^{1}$ and Hong-Hao Zhang ${ }^{1, \dagger}$ \\ ${ }^{1}$ School of Physics, Sun Yat-Sen University, Guangzhou 510275, China \\ ${ }^{2}$ Department of Physics, Yunnan University, Kunming 650091, China
}

(Received 28 July 2019; published 10 October 2019)

\begin{abstract}
We study a dark matter model with one singlet complex scalar and two Higgs doublets. The scalar potential respects a softly broken global symmetry, which makes the imaginary part of the singlet become a pseudo-Nambu-Goldstone boson acting as a dark matter candidate. The pseudo-Nambu-Goldstone nature of the boson leads to the vanishing of its tree-level scattering amplitude off nucleons at zero momentum transfer. Therefore, although the interaction strength could be sufficiently large to yield a viable relic abundance via thermal mechanism, direct detection is incapable of probing this candidate. We further investigate the constraints from Higgs measurements, relic abundance observation, and indirect detection.
\end{abstract}

DOI: 10.1103/PhysRevD.100.075011

\section{INTRODUCTION}

Astrophysical and cosmological observations suggest that the majority of matter in the present Universe consists of a nonluminous component called dark matter (DM). In the conventional paradigm, dark matter is a thermal relic remaining from the early Universe, implying that the interaction strength between DM and standard model (SM) particles may be comparable to the strength of weak interactions [1-3]. However, null signal results from recent direct detection experiments have put rather stringent constraints on the DM-nucleon scattering cross section [4-6]. This has become a great challenge to the thermal DM paradigm.

A natural way out is to suppress DM-nucleon scattering in direct detection experiments without suppressing DM annihilation in the early Universe. One possibility is that there are some blind spots with particular parameters leading to the suppression of the DM couplings relevant to direct detection [7-11]. Additionally, the relevant DM couplings could vanish due to special symmetries [12-17]. Moreover, DM-nucleon scattering mediated by pseudoscalars can evade direct detection constraints [18-24]. Furthermore, the DM-nucleon scattering amplitude could be greatly suppressed if the DM particle is a pseudoNambu-Goldstone boson (pNGB) protected by an approximate global symmetry [25-32].

\footnotetext{
yuzhaoh5@mail.sysu.edu.cn

zhh98@mail.sysu.edu.cn
}

Published by the American Physical Society under the terms of the Creative Commons Attribution 4.0 International license. Further distribution of this work must maintain attribution to the author(s) and the published article's title, journal citation, and DOI. Funded by SCOAP.
In the last case, tree-level interactions of a pNGB are generally momentum suppressed. As direct detection experiments essentially operate in the zero momentum transfer limit, the amplitude of pNGB dark matter scattering off nucleons vanishes at tree level [26]. Loop corrections could break the global symmetry, resulting in nonvanishing scattering. Nevertheless, further investigations have shown that the DM-nucleon cross section at oneloop level is rather small, far away from the capability of current direct detection experiments $[33,34]$. Therefore, such a pNGB DM framework seems very appealing for thermal DM.

Previous studies in this framework assumed that the Higgs sector just involves one Higgs doublet as in the SM [25-34]. In this work, we would like to extend the study to two Higgs doublets [35]. A Higgs sector with two SU(2) L doublets has fairly good motivations. First, two Higgs doublets are typically required for constructing realistic supersymmetric [36] and axion [37] models. Second, the flexible scalar mass spectrum and additional $C P$ violation sources in two-Higgs-doublet models may be helpful for generating a desired baryon asymmetry of the Universe through the baryogenesis mechanism [38]. Finally, two Higgs doublets could provide an available portal to thermal dark matter with attractive phenomenological features [11,18-21,23,24,39-43].

In this paper, we consider that the scalar sector involves two $\mathrm{SU}(2)_{\mathrm{L}}$ Higgs doublets as well as a complex scalar $S$, which is a SM gauge singlet. Most terms in the scalar potential obey a global U(1) symmetry $S \rightarrow e^{i \alpha} S$. The exception is a quadratic term that softly breaks this symmetry and gives mass to the imaginary part of $S$, denoted as $\chi$. The real scalar $\chi$ is what we call pNGB dark matter. Its pNGB nature makes its scattering amplitude off nucleons vanish at tree level, evading direct detection 
constraints. Nonetheless, it is able to obtain an observed DM relic abundance via the thermal production mechanism. We will perform a random scan in the parameter space to investigate reasonable parameter points that satisfy current Higgs measurements at the Large Hadron Collider (LHC), observation of the DM relic abundance, and constraints from indirect detection experiments.

The paper is organized as follows. In Sec. II, we describe the details of the pNGB DM model with two Higgs doublets, including the scalar potential, mass eigenstates, four types of Yukawa couplings, the vanishing of the DMnucleon scattering amplitude, and the alignment limit. In Sec. III, we perform a random scan in the parameter space and investigate phenomenological constraints from LHC Higgs measurements, relic abundance observation, and indirect detection. Section IV gives the conclusions and outlook. In the Appendix A, we write down the scalar and gauge trilinear couplings. Appendix B gives some expressions for decay widths of the SM-like Higgs boson.

\section{MODEL DETAILS}

In this section, we study the model details. As explained above, we assume that the scalar sector involves two Higgs doublets and one SM gauge singlet, and there is a softly broken global U(1) symmetry leading to pNGB dark matter. The fermion content is assumed to be the same as in the SM. Analogous to generic two-Higgs-doublet models, there are four types of Yukawa couplings that do not induce flavor-changing neutral currents (FCNCs) at tree level. We find that these four types are all applicable to our purpose.

\section{A. Scalar potential}

The two $\mathrm{SU}(2)_{\mathrm{L}}$ Higgs-doublet fields are denoted as $\Phi_{1}$ and $\Phi_{2}$, both carrying hypercharge $+1 / 2$. The complex scalar $S$ is a $\mathrm{SU}(2)_{\mathrm{L}}$ singlet and carries no hypercharge. For simplicity, we make two common assumptions for the scalar potential. The first assumption is that $C P$ is conserved in the scalar sector, leading to only real coefficients. The second one is that there is a $Z_{2}$ symmetry $\Phi_{1} \rightarrow-\Phi_{1}$ or $\Phi_{2} \rightarrow-\Phi_{2}$ forbidding quartic terms that are odd in either $\Phi_{1}$ or $\Phi_{2}$, but such a symmetry can be softly broken by quadratic terms.

Under these assumptions, the general terms in the scalar potential constructed with $\Phi_{1}$ and $\Phi_{2}$ are given by [35]

$$
\begin{aligned}
V_{1}= & m_{11}^{2}\left|\Phi_{1}\right|^{2}+m_{22}^{2}\left|\Phi_{2}\right|^{2}-m_{12}^{2}\left(\Phi_{1}^{\dagger} \Phi_{2}+\Phi_{2}^{\dagger} \Phi_{1}\right) \\
& +\frac{\lambda_{1}}{2}\left|\Phi_{1}\right|^{4}+\frac{\lambda_{2}}{2}\left|\Phi_{2}\right|^{4}+\lambda_{3}\left|\Phi_{1}\right|^{2}\left|\Phi_{2}\right|^{2}+\lambda_{4}\left|\Phi_{1}^{\dagger} \Phi_{2}\right|^{2} \\
& +\frac{\lambda_{5}}{2}\left[\left(\Phi_{1}^{\dagger} \Phi_{2}\right)^{2}+\left(\Phi_{2}^{\dagger} \Phi_{1}\right)^{2}\right] .
\end{aligned}
$$

And we can write down the potential terms that involve $S$ and respect a global U(1) symmetry $S \rightarrow e^{i \alpha} S$,
$V_{2}=-m_{S}^{2}|S|^{2}+\frac{\lambda_{S}}{2}|S|^{4}+\kappa_{1}\left|\Phi_{1}\right|^{2}|S|^{2}+\kappa_{2}\left|\Phi_{2}\right|^{2}|S|^{2}$.

In addition, we introduce a quadratic term softly breaking the global U(1) symmetry,

$$
V_{\text {soft }}=-\frac{m_{S}^{\prime 2}}{4} S^{2}+\text { H.c. }
$$

Note that even if $m_{S}^{\prime 2}$ is complex, we can always make it real and positive by a phase redefinition of $S$. Then $V_{2}$ and $V_{\text {soft }}$ respect a dark $C P$ symmetry $S \rightarrow S^{*}[26,31]$. The soft breaking term $V_{\text {soft }}$ can be justified by treating $m_{S}^{\prime 2}$ as a spurion, arising from a more fundamental theory that does not induce other soft breaking terms involving odd powers of $S[26,28]$.

Now the whole scalar potential is

$$
V=V_{1}+V_{2}+V_{\text {soft }} \text {. }
$$

In particular regions of the parameter space, $\Phi_{1}, \Phi_{2}$, and $S$ develop nonzero vacuum expectation values (VEVs) $v_{1}, v_{2}$, and $v_{s}$. They can be expanded as

$$
\begin{aligned}
\Phi_{1} & =\left(\begin{array}{c}
\phi_{1}^{+} \\
\left(v_{1}+\rho_{1}+i \eta_{1}\right) / \sqrt{2}
\end{array}\right), \\
\Phi_{2} & =\left(\begin{array}{c}
\phi_{2}^{+} \\
\left(v_{2}+\rho_{2}+i \eta_{2}\right) / \sqrt{2}
\end{array}\right), \\
S & =\frac{v_{s}+s+i \chi}{\sqrt{2}} .
\end{aligned}
$$

By minimizing the potential, we find the following stationary point conditions:

$$
\begin{aligned}
& m_{11}^{2}=\frac{v_{2}}{v_{1}} m_{12}^{2}-\frac{1}{2} \lambda_{1} v_{1}^{2}-\frac{1}{2} \lambda_{345} v_{2}^{2}-\frac{1}{2} \kappa_{1} v_{s}^{2} \\
& m_{22}^{2}=\frac{v_{1}}{v_{2}} m_{12}^{2}-\frac{1}{2} \lambda_{2} v_{2}^{2}-\frac{1}{2} \lambda_{345} v_{1}^{2}-\frac{1}{2} \kappa_{2} v_{s}^{2} \\
& m_{S}^{2}=-\frac{1}{2} m_{S}^{\prime 2}+\frac{1}{2} \lambda_{S} v_{s}^{2}+\frac{1}{2} \kappa_{1} v_{1}^{2}+\frac{1}{2} \kappa_{2} v_{2}^{2}
\end{aligned}
$$

where

$$
\lambda_{345} \equiv \lambda_{3}+\lambda_{4}+\lambda_{5}
$$

Note that all terms in $V_{2}$ and $V_{\text {soft }}$ are products of $|S|^{2}$ or of $S^{2}+\left(S^{*}\right)^{2}$. As their expansions are

$$
\begin{aligned}
|S|^{2} & =\frac{1}{2}\left(v_{s}^{2}+s^{2}+\chi^{2}\right)+v_{s} s, \\
S^{2}+\left(S^{*}\right)^{2} & =v_{s}^{2}+s^{2}-\chi^{2}+2 v_{s} s,
\end{aligned}
$$


the real scalar $\chi$ always appears in pair in the scalar potential. Therefore, $\chi$ cannot decay, becoming a stable DM candidate.

\section{B. Mass eigenstates}

After the scalar fields obtain their VEVs, the mass squared of $\chi$ is

$m_{\chi}^{2}=-m_{S}^{2}+\frac{1}{2} m_{S}^{\prime 2}+\frac{1}{2} \lambda_{S} v_{S}^{2}+\frac{1}{2} \kappa_{1} v_{1}^{2}+\frac{1}{2} \kappa_{2} v_{2}^{2}=m_{S}^{\prime 2}$,

where the terms with VEVs are totally canceled by the third stationary point condition (8). If $m_{S}^{\prime 2}=0$, there is no soft breaking term, and $\chi$ is a massless Nambu-Goldstone boson. If $m_{S}^{\prime 2}>0, \chi$ would have a physical mass $m_{\chi}=m_{S}^{\prime}$, behaving as a pseudo-Nambu-Goldstone boson. This is exactly what we want.

The mass terms for the charged scalars are derived as

$$
\begin{aligned}
-\mathcal{L}_{\text {mass }, \phi}= & {\left[m_{12}^{2}-\frac{1}{2}\left(\lambda_{4}+\lambda_{5}\right) v_{1} v_{2}\right]\left(\begin{array}{ll}
\phi_{1}^{-}, & \phi_{2}^{-}
\end{array}\right) } \\
& \times\left(\begin{array}{cc}
v_{2} / v_{1} & -1 \\
-1 & v_{1} / v_{2}
\end{array}\right)\left(\begin{array}{l}
\phi_{1}^{+} \\
\phi_{2}^{+}
\end{array}\right),
\end{aligned}
$$

while those for the $C P$-odd scalars are given by

$$
\begin{aligned}
-\mathcal{L}_{\text {mass }, \eta}= & \frac{1}{2}\left(m_{12}^{2}-\lambda_{5} v_{1} v_{2}\right)\left(\eta_{1}, \quad \eta_{2}\right) \\
& \times\left(\begin{array}{cc}
v_{2} / v_{1} & -1 \\
-1 & v_{1} / v_{2}
\end{array}\right)\left(\begin{array}{l}
\eta_{1} \\
\eta_{2}
\end{array}\right) .
\end{aligned}
$$

The above mass terms can be diagonalized by rotations

$$
\begin{aligned}
\left(\begin{array}{l}
\phi_{1}^{+} \\
\phi_{2}^{+}
\end{array}\right) & =R(\beta)\left(\begin{array}{l}
G^{+} \\
H^{+}
\end{array}\right), \\
\left(\begin{array}{l}
\eta_{1} \\
\eta_{2}
\end{array}\right) & =R(\beta)\left(\begin{array}{c}
G^{0} \\
a
\end{array}\right), \\
R(\beta) & =\left(\begin{array}{cc}
\cos \beta & -\sin \beta \\
\sin \beta & \cos \beta
\end{array}\right),
\end{aligned}
$$

where the rotation angle $\beta$ satisfies

$$
\tan \beta=\frac{v_{2}}{v_{1}} .
$$

Now $G^{ \pm}$and $G^{0}$ are massless Nambu-Goldstone bosons eaten by the weak gauge bosons $W^{ \pm}$and $Z$, while $H^{ \pm}$and $a$ are physical states with masses

$$
\begin{aligned}
m_{H^{+}}^{2} & =\frac{v_{1}^{2}+v_{2}^{2}}{v_{1} v_{2}}\left[m_{12}^{2}-\frac{1}{2}\left(\lambda_{4}+\lambda_{5}\right) v_{1} v_{2}\right], \\
m_{a}^{2} & =\frac{v_{1}^{2}+v_{2}^{2}}{v_{1} v_{2}}\left(m_{12}^{2}-\lambda_{5} v_{1} v_{2}\right) .
\end{aligned}
$$

The $C P$-even scalars $\rho_{1}, \rho_{2}$, and $s$ mix with each other. Their mass terms are

$$
-\mathcal{L}_{\text {mass }, \rho s}=\frac{1}{2}\left(\begin{array}{lll}
\rho_{1}, & \rho_{2}, & s
\end{array}\right) \mathcal{M}_{\rho s}^{2}\left(\begin{array}{c}
\rho_{1} \\
\rho_{2} \\
s
\end{array}\right),
$$

where the elements of the $3 \times 3$ symmetric mass-squared matrix $\mathcal{M}_{\rho s}^{2}$ are given by

$$
\begin{aligned}
& \left(\mathcal{M}_{\rho s}^{2}\right)_{11}=\lambda_{1} v_{1}^{2}+\frac{v_{2}}{v_{1}} m_{12}^{2}, \\
& \left(\mathcal{M}_{\rho s}^{2}\right)_{22}=\lambda_{2} v_{2}^{2}+\frac{v_{1}}{v_{2}} m_{12}^{2}, \\
& \left(\mathcal{M}_{\rho s}^{2}\right)_{33}=\lambda_{S} v_{s}^{2},
\end{aligned}
$$

$$
\begin{aligned}
& \left(\mathcal{M}_{\rho s}^{2}\right)_{12}=\lambda_{345} v_{1} v_{2}-m_{12}^{2}, \quad\left(\mathcal{M}_{\rho s}^{2}\right)_{13}=\kappa_{1} v_{1} v_{s}, \\
& \left(\mathcal{M}_{\rho s}^{2}\right)_{23}=\kappa_{2} v_{2} v_{s} .
\end{aligned}
$$

$\mathcal{M}_{\rho s}^{2}$ can be diagonalized by a $3 \times 3$ real orthogonal matrix $O$,

$$
O^{\mathrm{T}} \mathcal{M}_{\rho S}^{2} O=\operatorname{diag}\left(m_{h_{1}}^{2}, m_{h_{2}}^{2}, m_{h_{3}}^{2}\right) .
$$

The mass eigenstates $h_{i}(i=1,2,3)$ are then related to the interaction eigenstates by

$$
\left(\begin{array}{c}
\rho_{1} \\
\rho_{2} \\
s
\end{array}\right)=O\left(\begin{array}{c}
h_{1} \\
h_{2} \\
h_{3}
\end{array}\right) .
$$

One of $h_{i}$ should behave like the SM Higgs boson in order to be consistent with observation. Below we adopt a convention with $m_{h_{1}} \leq m_{h_{2}} \leq m_{h_{3}}$.

From the covariant kinetic terms

$$
\mathcal{L}_{\text {kin }}=\left(D^{\mu} \Phi_{1}\right)^{\dagger} D_{\mu} \Phi_{1}+\left(D^{\mu} \Phi_{2}\right)^{\dagger} D_{\mu} \Phi_{2},
$$

we derive the mass terms for the weak gauge bosons,

$$
\mathcal{L}_{\text {mass }, W Z}=\frac{g^{2}}{4}\left(v_{1}^{2}+v_{2}^{2}\right) W^{-, \mu} W_{\mu}^{+}+\frac{1}{2} \frac{g^{2}}{4 c_{\mathrm{W}}^{2}}\left(v_{1}^{2}+v_{2}^{2}\right) Z^{\mu} Z_{\mu},
$$

where $c_{\mathrm{W}} \equiv \cos \theta_{\mathrm{W}}$ with $\theta_{\mathrm{W}}$ denoting the Weinberg angle, and $g$ is the $\mathrm{SU}(2)_{\mathrm{L}}$ gauge coupling. Defining $v \equiv$ $\sqrt{v_{1}^{2}+v_{2}^{2}}$, the masses of $W$ and $Z$ bosons become 


$$
m_{W}=\frac{g v}{2}, \quad m_{Z}=\frac{g v}{2 c_{\mathrm{W}}},
$$

just as in the SM. From the Fermi constant $G_{\mathrm{F}}=g^{2} /$ $\left(4 \sqrt{2} m_{\mathrm{W}}^{2}\right)$, we obtain $v=\left(\sqrt{2} G_{\mathrm{F}}\right)^{-1 / 2}=246.22 \mathrm{GeV}$. Note that $v_{1}$ and $v_{2}$ satisfy $v_{1}=v c_{\beta}$ and $v_{2}=v s_{\beta}$, where we have used the shorthand notations $s_{\beta} \equiv \sin \beta$ and $c_{\beta} \equiv \cos \beta$.

The scalar and gauge trilinear couplings of the scalar mass eigenstates can be found in Appendix A.

\section{Yukawa couplings}

Unlike the standard model, Yukawa couplings between the two Higgs doublets and SM fermions generally lead to tree-level FCNCs, which could cause phenomenological problems in flavor physics. This is because diagonalizing the fermion mass matrix cannot make sure that the Yukawa interactions are also diagonalized. Nevertheless, if all fermions with the same quantum numbers just couple to the one same Higgs doublet, the FCNCs will be absent at tree level [35,44-46]. This can be achieved by assuming particular $Z_{2}$ symmetries for the Higgs doublets and fermions.

As a result, there are four independent types of Yukawa couplings without tree-level FCNCs, listed as follows.

Type I: $\mathcal{L}_{\mathrm{Y}, \mathrm{I}}=-y_{\ell_{i}} \bar{L}_{i \mathrm{~L}} \ell_{i \mathrm{R}} \Phi_{2}-\tilde{y}_{d}^{i j} \bar{Q}_{i \mathrm{~L}} d_{j \mathrm{R}}^{\prime} \Phi_{2}$

$$
-\tilde{y}_{u}^{i j} \bar{Q}_{i \mathrm{~L}} u_{j \mathrm{R}}^{\prime} \tilde{\Phi}_{2}+\text { H.c. }
$$

Type II : $\mathcal{L}_{\mathrm{Y}, \mathrm{II}}=-y_{\ell_{i}} \bar{L}_{i \mathrm{~L}} \ell_{i \mathrm{R}} \Phi_{1}-\tilde{y}_{d}^{i j} \bar{Q}_{i \mathrm{~L}} d_{j \mathrm{R}}^{\prime} \Phi_{1}$

$$
-\tilde{y}_{u}^{i j} \bar{Q}_{i \mathrm{~L}} u_{j \mathrm{R}}^{\prime} \tilde{\Phi}_{2}+\text { H.c. }
$$

Lepton specific: $\mathcal{L}_{\mathrm{Y}, \mathrm{L}}=-y_{\ell_{i}} \bar{L}_{i \mathrm{~L}} \ell_{i \mathrm{R}} \Phi_{1}-\tilde{y}_{d}^{i j} \bar{Q}_{i \mathrm{~L}} d_{j \mathrm{R}}^{\prime} \Phi_{2}$

$$
-\tilde{y}_{u}^{i j} \bar{Q}_{i \mathrm{~L}} u_{j \mathrm{R}}^{\prime} \tilde{\Phi}_{2}+\text { H.c. }
$$

Flipped: $\mathcal{L}_{\mathrm{Y}, \mathrm{F}}=-y_{\ell_{i}} \bar{L}_{i \mathrm{~L}} \ell_{i \mathrm{R}} \Phi_{2}-\tilde{y}_{d}^{i j} \bar{Q}_{i \mathrm{~L}} d_{j \mathrm{R}}^{\prime} \Phi_{1}$

$$
-\tilde{y}_{u}^{i j} \bar{Q}_{i \mathrm{~L}} u_{j \mathrm{R}}^{\prime} \tilde{\Phi}_{2}+\text { H.c. }
$$

Here $\tilde{\Phi}_{2} \equiv i \sigma^{2} \Phi_{2}^{*}, L_{i \mathrm{~L}} \equiv\left(\nu_{i \mathrm{~L}}, \ell_{i \mathrm{~L}}\right)^{\mathrm{T}}$, and $Q_{i \mathrm{~L}} \equiv\left(u_{i \mathrm{~L}}^{\prime}, d_{i \mathrm{~L}}^{\prime}\right)^{\mathrm{T}}$. The down-type and up-type quark Yukawa matrices $\tilde{y}_{d}^{i j}$ and $\tilde{y}_{u}^{i j}$ can be diagonalized through $\left(U_{d}\right)_{i j}^{\dagger} \tilde{y}_{d}^{j k}\left(U_{d}\right)_{k l}=y_{d_{i}} \delta_{i l}$ and $\left(U_{u}\right)_{i j}^{\dagger} \tilde{y}_{u}^{j k}\left(U_{u}\right)_{k l}=y_{u_{i}} \delta_{i l}$. Thus, the interaction eigenstates $u_{i}^{\prime}$ and $d_{i}^{\prime}$ are related to the mass eigenstates $u_{i}$ and $d_{i}$ via $d_{i}^{\prime}=\left(U_{d}\right)_{i j} d_{j}$ and $u_{i}^{\prime}=\left(U_{u}\right)_{i j} u_{j}$. The CabibboKobayashi-Maskawa matrix is defined as $V_{i j} \equiv\left(U_{u}\right)_{i k}^{\dagger}$ $\left(U_{d}\right)_{k j}$. As we would not discuss neutrino physics in this work, we assume the lepton sector is the same as in the SM.

After the scalars develop the VEVs, the Yukawa interactions provide mass terms to the fermions. For the mass eigenstates, the four types of Yukawa terms can be expressed in the same form,
TABLE I. Coefficients $\xi_{h_{i}}^{f}$ and $\xi_{a}^{f}$ in the four types of Yukawa couplings.

\begin{tabular}{lcccc}
\hline \hline & Type I & Type II & Lepton specific & Flipped \\
\hline$\xi_{h_{i}}^{\ell_{j}}$ & $O_{2 i} / \sin \beta$ & $O_{1 i} / \cos \beta$ & $O_{1 i} / \cos \beta$ & $O_{2 i} / \sin \beta$ \\
$\xi_{h_{j}}^{d_{j}}$ & $O_{2 i} / \sin \beta$ & $O_{1 i} / \cos \beta$ & $O_{2 i} / \sin \beta$ & $O_{1 i} / \cos \beta$ \\
$\xi_{h_{i}}^{u_{j}}$ & $O_{2 i} / \sin \beta$ & $O_{2 i} / \sin \beta$ & $O_{2 i} / \sin \beta$ & $O_{2 i} / \sin \beta$ \\
$\xi_{a}^{\ell_{j}}$ & $\cot \beta$ & $-\tan \beta$ & $-\tan \beta$ & $\cot \beta$ \\
$\xi_{a}^{d_{j}}$ & $\cot \beta$ & $-\tan \beta$ & $\cot \beta$ & $-\tan \beta$ \\
$\xi_{a}^{u_{j}}$ & $-\cot \beta$ & $-\cot \beta$ & $-\cot \beta$ & $-\cot \beta$ \\
\hline \hline
\end{tabular}

$$
\begin{aligned}
\mathcal{L}_{\mathrm{Y}}= & \sum_{f=\ell_{j}, d_{j}, u_{j}}\left[-m_{f} \bar{f} f-\frac{m_{f}}{v}\left(\sum_{i=1}^{3} \xi_{h_{i}}^{f} h_{i} \bar{f} f+\xi_{a}^{f} a \bar{f} i \gamma_{5} f\right)\right] \\
& -\frac{\sqrt{2}}{v}\left[H ^ { + } \left(\xi_{a}^{\ell_{i}} m_{\ell_{i}} \bar{\nu}_{i} P_{\mathrm{R}} \ell_{i}+\xi_{a}^{d_{j}} m_{d_{j}} V_{i j} \bar{u}_{i} P_{\mathrm{R}} d_{j}\right.\right. \\
& \left.\left.+\xi_{a}^{u_{i}} m_{u_{i}} V_{i j} \bar{u}_{i} P_{\mathrm{L}} d_{j}\right)+ \text { H.c. }\right],
\end{aligned}
$$

where $P_{\mathrm{L}}$ and $P_{\mathrm{R}}$ are the left- and right-handed projection operators, respectively. The coefficients $\xi_{h_{i}}^{f}$ and $\xi_{a}^{f}$ are listed in Table I.

\section{Vanishing of the DM-nucleon scattering amplitude}

In this subsection, we verify that the tree-level amplitude of DM scattering off nucleons vanishes at zero momentum transfer. In our case, DM-nucleon scattering is induced by DM-quark scattering. Therefore, we just need to prove that the DM-quark scattering amplitude vanishes in the zero momentum transfer limit.

From the $U(1)$ symmetric potential (2), we obtain the trilinear couplings for the DM candidate $\chi$ as

$\mathcal{L}_{\text {tri } \chi^{2}}=-\frac{1}{2}\left(\kappa_{1} v_{1} \rho_{1}+\kappa_{2} v_{2} \rho_{2}+\lambda_{S} v_{s} s\right) \chi^{2}=\frac{1}{2} \sum_{i=1}^{3} g_{h_{i} \chi^{2}} h_{i} \chi^{2}$,

where the coupling coefficients for the mass eigenstates are given by

$$
g_{h_{i} \chi^{2}}=-\kappa_{1} v_{1} O_{1 i}-\kappa_{2} v_{2} O_{2 i}-\lambda_{S} v_{s} O_{3 i} .
$$

At tree level, only the $C P$-even Higgs bosons $h_{1}, h_{2}$, and $h_{3}$ can mediate $\chi$ scattering off quarks. The Feynman diagram is shown in Fig. 1.

Take the type-I Yukawa couplings as an example. Defining a Lorentz invariant $t \equiv p_{\mu} p^{\mu}$, where $p^{\mu}$ is the 4-momentum of the mediator $h_{i}$, we can write down the DM-quark scattering amplitude as 


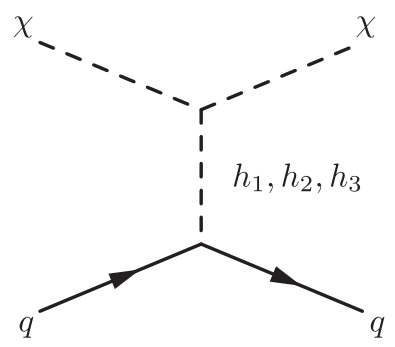

FIG. 1. Feynman diagram for DM-quark scattering.

$$
\begin{aligned}
i \mathcal{M}= & \frac{m_{q}}{v s_{\beta}} \bar{u}\left(k_{2}\right) u\left(k_{1}\right)\left(g_{h_{1} \chi^{2}} \frac{i}{t-m_{h_{1}}^{2}} O_{21}+g_{h_{2} \chi^{2}} \frac{i}{t-m_{h_{2}}^{2}} O_{22}\right. \\
& \left.+g_{h_{3} \chi^{2}} \frac{i}{t-m_{h_{3}}^{2}} O_{23}\right),
\end{aligned}
$$

where $u\left(k_{1}\right)$ and $\bar{u}\left(k_{2}\right)$ are the wave functions for the incoming and outgoing quarks, respectively. In the zero momentum transfer limit, $t \rightarrow 0$, and the above amplitude can be reexpressed as

$$
\begin{aligned}
i \mathcal{M} \rightarrow & -i \frac{m_{q}}{v s_{\beta}} \bar{u}\left(k_{2}\right) u\left(k_{1}\right)\left(g_{h_{1} \chi^{2}}, \quad g_{h_{2} \chi^{2}}, \quad g_{h_{3} \chi^{2}}\right) \\
& \times\left(\mathcal{M}_{h}^{2}\right)^{-1} O^{\mathrm{T}}\left(\begin{array}{l}
0 \\
1 \\
0
\end{array}\right),
\end{aligned}
$$

where $\left(\mathcal{M}_{h}^{2}\right)^{-1}=\operatorname{diag}\left(m_{h_{1}}^{-2}, m_{h_{2}}^{-2}, m_{h_{3}}^{-2}\right)$ is the inverse of the diagonalized mass-squared matrix $\mathcal{M}_{h}^{2} \equiv \operatorname{diag}\left(m_{h_{1}}^{2}\right.$, $m_{h_{2}}^{2}, m_{h_{3}}^{2}$ ). From Eqs. (31) and (20), we have

$$
\begin{aligned}
\left(g_{h_{1} \chi^{2}}, \quad g_{h_{2} \chi^{2}}, \quad g_{h_{3} \chi^{2}}\right) & =-\left(\kappa_{1} v_{1}, \quad \kappa_{2} v_{2}, \quad \lambda_{s} v_{s}\right) O \\
\left(\mathcal{M}_{h}^{2}\right)^{-1} & =O^{\mathrm{T}}\left(\mathcal{M}_{\rho s}^{2}\right)^{-1} O
\end{aligned}
$$

Utilizing these equations as well as the orthogonality of $O$, we obtain

$$
\begin{aligned}
i \mathcal{M} \rightarrow & i \frac{m_{q}}{v s_{\beta}} \bar{u}\left(k_{2}\right) u\left(k_{1}\right)\left(\kappa_{1} v_{1}, \quad \kappa_{2} v_{2}, \quad \lambda_{S} v_{s}\right) \\
& \times\left(\mathcal{M}_{\rho s}^{2}\right)^{-1}\left(\begin{array}{l}
0 \\
1 \\
0
\end{array}\right) .
\end{aligned}
$$

This can be understood as the amplitude expressed in the interaction basis [26].

The inverse of $\mathcal{M}_{\rho s}^{2}$ can be expressed as its adjugate $\mathcal{A}$ divided by its determinant, i.e., $\left(\mathcal{M}_{\rho s}^{2}\right)^{-1}=\mathcal{A} / \operatorname{det}\left(\mathcal{M}_{\rho s}^{2}\right)$. The relevant elements of $\mathcal{A}$ are

$$
\mathcal{A}_{12}=-\left(\lambda_{345} v_{1} v_{2}-m_{12}^{2}\right) \lambda_{S} v_{s}^{2}+\kappa_{1} \kappa_{2} v_{1} v_{2} v_{s}^{2}
$$

$$
\begin{gathered}
\mathcal{A}_{22}=\left(\lambda_{1} v_{1}^{2}+m_{12}^{2} \tan \beta\right) \lambda_{s} v_{s}^{2}-\kappa_{1}^{2} v_{1}^{2} v_{s}^{2}, \\
\mathcal{A}_{32}=-\left(\lambda_{1} v_{1}^{2}+m_{12}^{2} \tan \beta\right) \kappa_{2} v_{2} v_{s}+\left(\lambda_{345} v_{1} v_{2}-m_{12}^{2}\right) \kappa_{1} v_{1} v_{s} .
\end{gathered}
$$

We then have

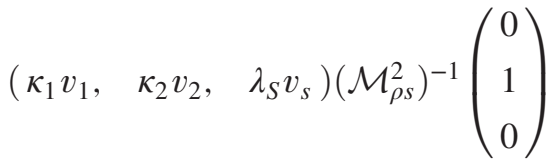

$$
\begin{aligned}
& =\operatorname{det}^{-1}\left(\mathcal{M}_{\rho s}^{2}\right)\left(\kappa_{1} v_{1} \mathcal{A}_{12}+\kappa_{2} v_{2} \mathcal{A}_{22}+\lambda_{S} v_{s} \mathcal{A}_{32}\right) \\
& =0 \text {. }
\end{aligned}
$$

Therefore, we have proven that the tree-level DM-quark amplitude $i \mathcal{M}$ vanishes in the zero momentum transfer limit for the type-I Yukawa couplings. Similarly, we can prove this for the type-II, lepton-specific, and flipped Yukawa couplings.

As the global U(1) symmetry is softly broken, loop corrections would give a nonvanishing DM-nucleon scattering cross section [26]. Nonetheless, we expect that the loopinduced cross section should be typically $\lesssim \mathcal{O}\left(10^{-50}\right) \mathrm{cm}^{2}$, as suggested by the one-loop evaluation in Ref. [33] where only one Higgs doublet is considered. Thus, current and near future direct detection experiments should not be able to probe our pNGB DM model.

\section{E. Alignment limit}

Current LHC Higgs measurements favor a $125 \mathrm{GeV}$ SM-like Higgs boson. If one of the $C P$-even Higgs bosons mimics the SM Higgs boson, the constraints from Higgs measurements can be easily satisfied. For the two Higgs doublets, such a situation can be achieved by requiring that the additional scalars are much heavier than the weak scale so that the lightest $C P$-even Higgs boson reproduces SM-like Higgs signals at the LHC. This is known as the "decoupling" limit [47]. In general, a particular parameter set or relation leading to a $C P$-even Higgs boson mimicking the SM Higgs boson is referred as an "alignment" limit. The decoupling limit is of course an alignment limit, but it is less interesting, as the new particles might be too heavy to be accessed at the LHC.

A more interesting possibility is alignment without decoupling [48,49]. In order to find such a possibility, we may rotate the two Higgs doublets $\Phi_{1}$ and $\Phi_{2}$ into the Higgs basis [50,51]

$$
\left(\begin{array}{c}
\Phi_{h} \\
\Phi_{H}
\end{array}\right) \equiv R^{-1}(\beta)\left(\begin{array}{c}
\Phi_{1} \\
\Phi_{2}
\end{array}\right),
$$

and have 


$$
\begin{aligned}
\Phi_{h} & =\left(\begin{array}{c}
G^{+} \\
\left(v+h+i G^{0}\right) / \sqrt{2}
\end{array}\right), \\
\Phi_{H} & =\left(\begin{array}{c}
H^{+} \\
(H+i a) / \sqrt{2}
\end{array}\right) .
\end{aligned}
$$

Now $\Phi_{h}$ gains a VEV $v$ and contains a $C P$-even scalar $h$ as well as the Nambu-Goldstone bosons, while $\Phi_{H}$ has zero VEV and contains a $C P$-even scalar $H$ and the physical states $H^{+}$and $a$. Consequently, the tree-level interactions of the $C P$-even scalar $h$ with weak gauge bosons and SM fermions are totally identical to those of the Higgs boson in the SM. Therefore, the alignment limit means that $h$ does not mix with $H$ and $s$.

In the Higgs basis, the potential terms (1) transform to

$$
\begin{aligned}
V_{1}= & m_{h h}^{2}\left|\Phi_{h}\right|^{2}+m_{H H}^{2}\left|\Phi_{H}\right|^{2}-m_{h H}^{2}\left(\Phi_{h}^{\dagger} \Phi_{H}+\Phi_{H}^{\dagger} \Phi_{h}\right) \\
& +\frac{\lambda_{h}}{2}\left|\Phi_{h}\right|^{4}+\frac{\lambda_{H}}{2}\left|\Phi_{H}\right|^{4}+\tilde{\lambda}_{3}\left|\Phi_{h}\right|^{2}\left|\Phi_{H}\right|^{2} \\
& +\tilde{\lambda}_{4}\left|\Phi_{h}^{\dagger} \Phi_{H}\right|^{2}+\frac{1}{2}\left[\tilde{\lambda}_{5}\left(\Phi_{h}^{\dagger} \Phi_{H}\right)^{2}+\tilde{\lambda}_{6}\left|\Phi_{h}\right|^{2} \Phi_{H}^{\dagger} \Phi_{h}\right. \\
& \left.+\tilde{\lambda}_{7}\left|\Phi_{H}\right|^{2} \Phi_{h}^{\dagger} \Phi_{H}+\text { H.c. }\right],
\end{aligned}
$$

where the new parameters are related to the previous parameters by [42]

$$
\begin{gathered}
m_{h h}^{2}=c_{\beta}^{2} m_{11}^{2}+s_{\beta}^{2} m_{22}^{2}-2 s_{\beta} c_{\beta} m_{12}^{2}, \\
m_{H H}^{2}=s_{\beta}^{2} m_{11}^{2}+c_{\beta}^{2} m_{22}^{2}+2 s_{\beta} c_{\beta} m_{12}^{2}, \\
m_{h H}^{2}=s_{\beta} c_{\beta}\left(m_{11}^{2}-m_{22}^{2}\right)+\left(c_{\beta}^{2}-s_{\beta}^{2}\right) m_{12}^{2}, \\
\lambda_{h}=c_{\beta}^{4} \lambda_{1}+s_{\beta}^{4} \lambda_{2}+2 s_{\beta}^{2} c_{\beta}^{2} \lambda_{345}, \\
\lambda_{H}=s_{\beta}^{4} \lambda_{1}+c_{\beta}^{4} \lambda_{2}+2 s_{\beta}^{2} c_{\beta}^{2} \lambda_{345}, \\
\tilde{\lambda}_{3}=s_{\beta}^{2} c_{\beta}^{2}\left(\lambda_{1}+\lambda_{2}-2 \lambda_{4}-2 \lambda_{5}\right)+\left(s_{\beta}^{4}+c_{\beta}^{4}\right) \lambda_{3}, \\
\tilde{\lambda}_{4}=s_{\beta}^{2} c_{\beta}^{2}\left(\lambda_{1}+\lambda_{2}-2 \lambda_{3}-2 \lambda_{5}\right)+\left(s_{\beta}^{4}+c_{\beta}^{4}\right) \lambda_{4}, \\
\tilde{\lambda}_{5}=s_{\beta}^{2} c_{\beta}^{2}\left(\lambda_{1}+\lambda_{2}-2 \lambda_{3}-2 \lambda_{4}\right)+\left(s_{\beta}^{4}+c_{\beta}^{4}\right) \lambda_{5}, \\
\tilde{\lambda}_{6}=-s_{2 \beta}\left(c_{\beta}^{2} \lambda_{1}-s_{\beta}^{2} \lambda_{2}\right)+s_{2 \beta} c_{2 \beta} \lambda_{345}, \\
\tilde{\lambda}_{7}=-s_{2 \beta}\left(s_{\beta}^{2} \lambda_{1}-c_{\beta}^{2} \lambda_{2}\right)-s_{2 \beta} c_{2 \beta} \lambda_{345} .
\end{gathered}
$$

On the other hand, the potential terms (2) transform to

$$
\begin{aligned}
V_{2}= & -m_{S}^{2}|S|^{2}+\frac{\lambda_{S}}{2}|S|^{4}+\tilde{\kappa}_{1}\left|\Phi_{h}\right|^{2}|S|^{2}+\tilde{\kappa}_{2}\left|\Phi_{H}\right|^{2}|S|^{2} \\
& +\tilde{\kappa}_{3}\left(\Phi_{h}^{\dagger} \Phi_{H}+\Phi_{H}^{\dagger} \Phi_{h}\right)|S|^{2},
\end{aligned}
$$

where the new parameters are given by

$$
\begin{aligned}
& \tilde{\kappa}_{1}=c_{\beta}^{2} \kappa_{1}+s_{\beta}^{2} \kappa_{2}, \quad \tilde{\kappa}_{2}=s_{\beta}^{2} \kappa_{1}+c_{\beta}^{2} \kappa_{2}, \\
& \tilde{\kappa}_{3}=-s_{\beta} c_{\beta}\left(\kappa_{1}-\kappa_{2}\right) .
\end{aligned}
$$

Then the stationary point conditions for the scalar potential are

$$
\begin{aligned}
m_{h h}^{2} & =-\frac{1}{2} \lambda_{h} v^{2}-\frac{1}{2} \tilde{\kappa}_{1} v_{s}^{2}, \\
m_{h H}^{2} & =\frac{1}{4} \tilde{\lambda}_{6} v^{2}+\frac{1}{2} \tilde{\kappa}_{3} v_{s}^{2}, \\
m_{S}^{2} & =-\frac{1}{2} m_{S}^{\prime 2}+\frac{1}{2} \lambda_{S} v_{s}^{2}+\frac{1}{2} \tilde{\kappa}_{1} v^{2} .
\end{aligned}
$$

As a result, the mass-squared matrix for $C P$-even scalars $(h, H, s)$ is

$$
\mathcal{M}_{h H s}^{2}=\left(\begin{array}{ccc}
\lambda_{h} v^{2} & \tilde{\lambda}_{6} v^{2} / 2 & \tilde{\kappa}_{1} v v_{s} \\
\tilde{\lambda}_{6} v^{2} / 2 & m_{H H}^{2}+\left(\tilde{\lambda}_{345} v^{2}+\tilde{\kappa}_{2} v_{s}^{2}\right) / 2 & \tilde{\kappa}_{3} v v_{s} \\
\tilde{\kappa}_{1} v v_{s} & \tilde{\kappa}_{3} v v_{s} & \lambda_{S} v_{s}^{2}
\end{array}\right) .
$$

In order to prevent $h-H$ and $h$-s mixings, the off-diagonal terms $\left(\mathcal{M}_{h H s}^{2}\right)_{12}$ and $\left(\mathcal{M}_{h H s}^{2}\right)_{13}$ should be absent, corresponding to

$$
\tilde{\lambda}_{6}=\tilde{\kappa}_{1}=0 .
$$

This is the alignment condition in our model. When this condition is satisfied, the tree-level couplings of $h$ to SM particles are exactly the same as those of the SM Higgs boson.

\section{PHENOMENOLOGICAL CONSTRAINTS}

In this section, we take the type-I Yukawa couplings as an illuminating example to investigate the phenomenological constraints from Higgs measurements, relic abundance observation, and indirect detection.

\section{A. Parameter scan and Higgs measurements}

There are 12 free parameters in the model, which can be chosen as

$v_{s}, \quad m_{\chi}, \quad m_{12}^{2}, \quad \tan \beta, \quad \lambda_{1}, \quad \lambda_{2}, \quad \lambda_{3}, \quad \lambda_{4}$,

$\lambda_{5}, \lambda_{S}, \kappa_{1}, \kappa_{2}$.

In order to investigate the vast parameter space, we carry out a random scan within the following ranges:

$$
10 \mathrm{GeV}<v_{s}<10^{3} \mathrm{GeV}, \quad 10 \mathrm{GeV}<m_{\chi}<10^{4} \mathrm{GeV},
$$

$$
(10 \mathrm{GeV})^{2}<\left|m_{12}^{2}\right|<\left(10^{3} \mathrm{GeV}\right)^{2}, \quad 10^{-2}<\tan \beta<10^{2},
$$




$$
\begin{aligned}
& 10^{-3}<\lambda_{1}, \lambda_{2}, \lambda_{S}<1, \\
& 10^{-3}<\left|\lambda_{3}\right|,\left|\lambda_{4}\right|,\left|\lambda_{5}\right|,\left|\kappa_{1}\right|,\left|\kappa_{2}\right|<1 .
\end{aligned}
$$

Then we require the selected parameter points must give positive $m_{h_{1,2,3}}^{2}, m_{H^{+}}^{2}$, and $m_{a}^{2}$, ensuring physical scalar masses. Moreover, one of the $C P$-even Higgs bosons $h_{i}$ should have a mass within the $3 \sigma$ range of the measured SM-like Higgs boson mass $m_{h}=125.18 \pm 0.16 \mathrm{GeV}$ [52]. We recognize this scalar as the SM-like Higgs boson and denote it as $h_{\mathrm{SM}}$, and further examine if its properties are consistent with current measurements.

In the $\kappa$ framework [53], the couplings of the SM-like Higgs boson to SM particles can be expressed as

$$
\begin{aligned}
\mathcal{L}_{h_{\mathrm{SM}}}= & \kappa_{W} g m_{W} h_{\mathrm{SM}} W_{\mu}^{+} W^{-, \mu}+\kappa_{Z} \frac{g m_{Z}}{2 c_{\mathrm{W}}} h_{\mathrm{SM}} Z_{\mu} Z^{\mu} \\
& -\sum_{f} \kappa_{f} \frac{m_{f}}{v} h_{\mathrm{SM}} \bar{f} f+\kappa_{g} g_{h g g}^{\mathrm{SM}} h_{\mathrm{SM}} G_{\mu \nu}^{a} G^{a \mu \nu} \\
& +\kappa_{\gamma} g_{h \gamma \gamma}^{\mathrm{SM}} h_{\mathrm{SM}} A_{\mu \nu} A^{\mu \nu}+\kappa_{Z \gamma} g_{Z \gamma \gamma}^{\mathrm{SM}} h_{\mathrm{SM}} A_{\mu \nu} Z^{\mu \nu},
\end{aligned}
$$

where $g_{h g g}^{\mathrm{SM}}, g_{h \gamma \gamma}^{\mathrm{SM}}$, and $g_{Z \gamma \gamma}^{\mathrm{SM}}$ are the loop-induced effective couplings to $g g, \gamma \gamma$, and $Z \gamma$, respectively. $\kappa$ 's are coupling modifiers, whose values are all equal to 1 in the SM. Equation (A6) implies that $\kappa_{W}$ and $\kappa_{Z}$ are equal in our model, and we will use $\kappa_{V}$ representing both of them. Assuming the SM-like Higgs boson is $h_{\mathrm{SM}}=h_{i}$, we have

$$
\kappa_{V}=c_{\beta} O_{1 i}+s_{\beta} O_{2 i} .
$$

The coupling modifiers for fermions can be read off from Table I. For the type-I Yukawa couplings, all SM fermions have the same coupling modifier, given by

$$
\kappa_{f}=\frac{O_{2 i}}{s_{\beta}}
$$

It is also helpful to define another modifier $\kappa_{H}$ as

$$
\kappa_{H}^{2} \equiv \frac{\Gamma_{h_{\mathrm{SM}}}-\Gamma_{h_{\mathrm{SM}}}^{\mathrm{BSM}}}{\Gamma_{h}^{\mathrm{SM}}},
$$

where $\Gamma_{h}^{\mathrm{SM}}$ is the Higgs total decay width in the SM, $\Gamma_{h_{\mathrm{SM}}}$ is the total decay width of the SM-like Higgs boson $h_{\mathrm{SM}}$, and $\Gamma_{h_{\mathrm{SM}}}^{\mathrm{BSM}}$ is the $h_{\mathrm{SM}}$ decay width into final states beyond the SM (BSM). Thus, $\kappa_{H}$ indicates the deviation of the Higgs width decaying into SM final states and is also equal to 1 in the SM. In our model, $\Gamma_{h_{\mathrm{SM}}}^{\mathrm{BSM}}$ can be generally separated into two parts,

$$
\Gamma_{h_{\mathrm{SM}}}^{\mathrm{BSM}}=\Gamma_{h_{\mathrm{SM}}}^{\mathrm{inv}}+\Gamma_{h_{\mathrm{SM}}}^{\text {und }} .
$$

$\Gamma_{h_{\mathrm{SM}}}^{\mathrm{inv}}$ is the $h_{\mathrm{SM}}$ decay width into the invisible final state, i.e., a pair of the DM candidate $\chi$. $\Gamma_{h_{\mathrm{SM}}}^{\mathrm{und}}$ involves decay widths into all kinematically allowed BSM final states that are undetected in current LHC searches. Such final states may include $a a, H^{+} H^{-}, h_{i} h_{j}, a Z$, and $H^{ \pm} W^{\mp}$. The expressions for these decay widths are listed in Appendix B. Once all the decay widths are evaluated, we can determine the invisible and undetected BSM branching ratios via $\mathrm{BR}_{\mathrm{inv}}=\Gamma_{h_{\mathrm{SM}}}^{\mathrm{inv}} / \Gamma_{h_{\mathrm{SM}}}$ and $\mathrm{BR}_{\mathrm{und}}=$ $\Gamma_{h_{\mathrm{SM}}}^{\text {und }} / \Gamma_{h_{\mathrm{SM}}}$, respectively.

We utilize a numerical tool Lilith 1.1.4 [54] to study the constraints from current Higgs measurements. Lilith is able to construct an approximate likelihood based on experimental results of Higgs signal strength measurements. For each selected parameter point in our random scan, we put the corresponding $m_{h_{\mathrm{SM}}}, \kappa_{V}, \kappa_{f}, \mathrm{BR}_{\text {inv }}$, and $\mathrm{BR}_{\text {und }}$ into Lilith. Then Lilith can evaluate $\kappa_{g}, \kappa_{\gamma}$, and $\kappa_{Z \gamma}$ involving the loop contributions from SM fermions and gauge bosons whose couplings are modified by $\kappa_{f}$ and $\kappa_{V}$, including nextto-leading-order QCD corrections. Such an evaluation has neglected the loop contributions from the BSM scalars in our model. Nonetheless, these scalars are typically heavy and/or have small couplings. Therefore, their contributions are insignificant for most of the selected parameter points.

We further use Lilith to calculate the likelihood $-2 \ln L$ for each parameter point based on Tevatron data [55], ATLAS run 1 data [56-63], CMS run 1 data [64-68], ATLAS run 2 data [69-77], and CMS run 2 data [78-84]. We then transform $-2 \ln L$ to a $p$-value and require that the selected parameter points should give $p$-values larger than 0.05 . This means that we have rejected the parameter points that are excluded by data at $95 \%$ confidence level (C.L.).

Now we can analyze the properties of the remaining parameter points. Figure 2 shows the Lilith $p$-values of the selected parameter points projected in the $\tan \beta-\lambda_{1}$ and $\tan \beta-\lambda_{2}$ planes. We find that when $\tan \beta \lesssim 0.2(\tan \beta \gtrsim 5)$, $\lambda_{1}\left(\lambda_{2}\right)$ tends to converge on $\lambda_{\mathrm{SM}}=m_{h}^{2} / v^{2} \simeq 0.26$, which is the quartic Higgs coupling in the SM. This is because $\tan \beta \ll 1(\tan \beta \gg 1)$ leads to $v_{1} \gg v_{2}\left(v_{2} \gg v_{1}\right)$ and $\Phi_{1} \simeq \Phi_{h}\left(\Phi_{2} \simeq \Phi_{h}\right)$, i.e., $\Phi_{1}\left(\Phi_{2}\right)$ acting as the SM-like Higgs doublet. Since experimental data favor a SM-like Higgs boson, the corresponding quartic coupling would be close to its SM counterpart.

Additionally, we project the parameter points in the $m_{h_{\mathrm{SM}}}-m_{h_{1}}$ and $m_{h_{2}}-m_{h_{3}}$ planes in Figs. 3(a) and 3(b), respectively. In Fig. 3(a), the points with $h_{\mathrm{SM}}=h_{1}$ align along a horizontal line with $m_{h_{1}} \simeq 125 \mathrm{GeV}$, while the remaining points indicate that the SM-like Higgs boson is not the lightest $C P$-even Higgs boson $h_{1}$. On the other hand, two sets of aligned points in Fig. 3(b) correspond to $h_{\mathrm{SM}}=h_{2}$ and $h_{\mathrm{SM}}=h_{3}$.

The projection on the $m_{H^{+}} m_{a}$ plane is presented in Fig. 4. From Eq. (16), we know that the difference between the masses of the charged Higgs boson $H^{+}$and the $C P$-odd Higgs boson $a$ are due to the $\lambda_{4}$ and $\lambda_{5}$ couplings. If $m_{12}^{2}$ is much larger than the $\lambda_{4}$ and $\lambda_{5}$ contributions, the difference would be negligible, as demonstrated in Fig. 4 for $m_{H^{+}}, m_{a} \gtrsim 500 \mathrm{GeV}$. 


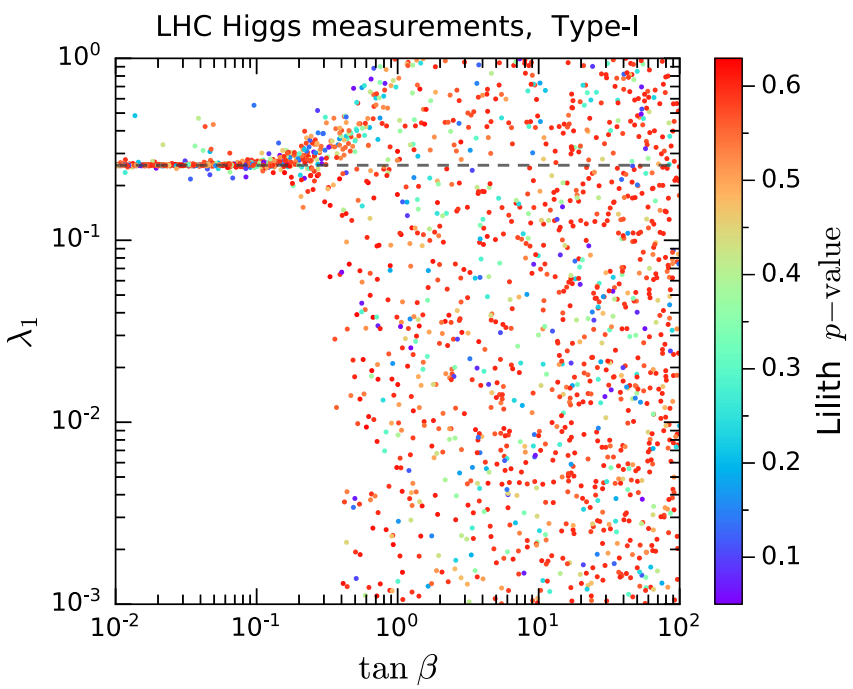

(a) $\tan \beta-\lambda_{1}$ plane.

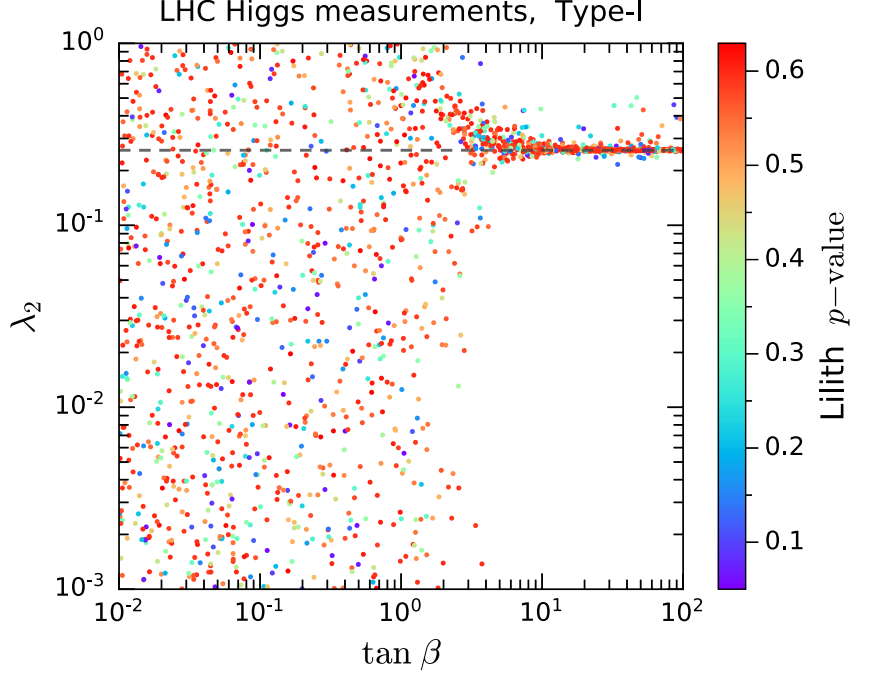

(b) $\tan \beta-\lambda_{2}$ plane.

FIG. 2. Lilith $p$-values for the selected parameter points projected in the $\tan \beta-\lambda_{1}$ (a) and $\tan \beta-\lambda_{2}$ (b) planes. The dashed lines indicate the value of $\lambda_{\mathrm{SM}}$.

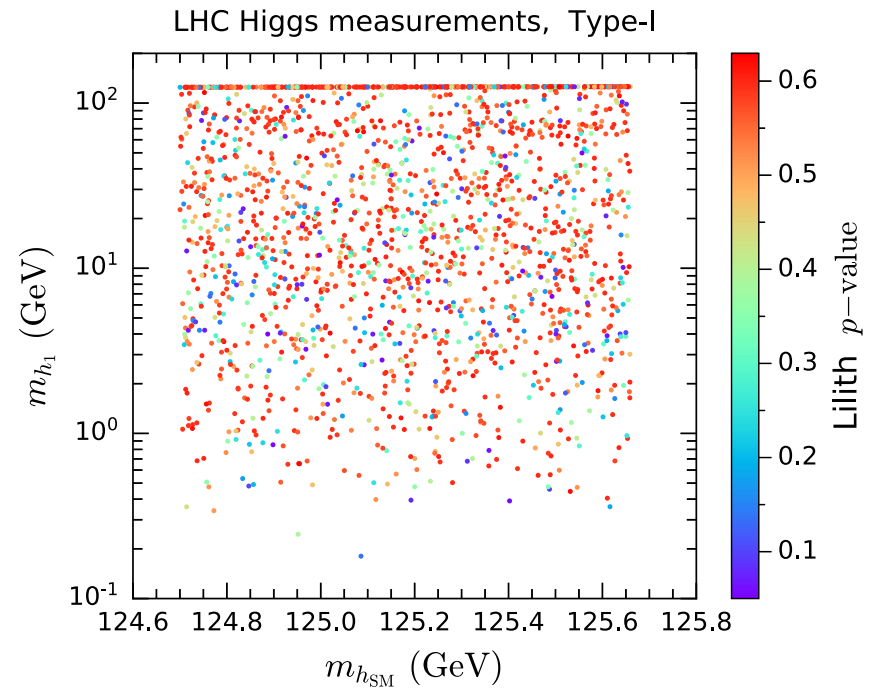

(a) $m_{h_{\mathrm{SM}}}-m_{h_{1}}$ plane.

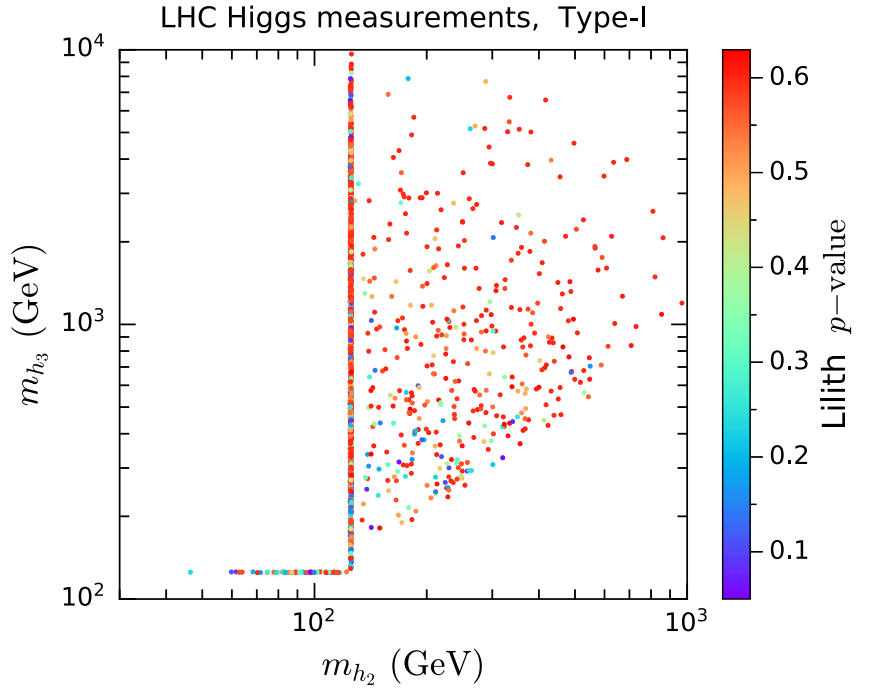

(b) $m_{h_{2}}-m_{h_{3}}$ plane.

FIG. 3. Lilith $p$-values for the selected parameter points projected in the $m_{h_{\mathrm{SM}}}-m_{h_{1}}$ (a) and $m_{h_{2}}-m_{h_{3}}$ (b) planes.

Figure 5(a) shows the projection on the $\left|\kappa_{V}\right|-\left|\kappa_{f}\right|$ plane. We find that the parameter points with $\left|\kappa_{V}\right| \simeq\left|\kappa_{f}\right| \simeq 1$ have the largest $p$-values, implying that current data still favor that the $125 \mathrm{GeV}$ Higgs boson has SM-like couplings. Nonetheless, $\left|\kappa_{V}\right|$ may range from $\sim 0.85$ to $\sim 1$, and $\left|\kappa_{f}\right|$ may range from $\sim 0.6$ to $\sim 1.3$. In addition, there are two categories of parameter points approximately aligning along two outstanding lines.

Category 1: One line in Fig. 5(a) corresponds to $\left|\kappa_{V}\right| \simeq\left|\kappa_{f}\right|$. Actually, the signs of $\kappa_{V}$ and $\kappa_{f}$ are the same for all selected parameter points. This line is thus related to $\kappa_{V} \simeq \kappa_{f}$. The main reason is that if $\tan \beta \gg 1$, we have $s_{\beta} \simeq 1$ and $c_{\beta} \simeq 0$, and Eqs. (59) and (60) become $\kappa_{V} \simeq \kappa_{f} \simeq O_{2 i}$, where $\kappa_{V}$ and $\kappa_{f}$ have a nearly total positive correlation. As $\left|O_{2 i}\right| \leq 1$, in this case both $\left|\kappa_{V}\right|$ and $\left|\kappa_{f}\right|$ cannot exceed one. Most of the parameter points in this category correspond to the horizontal line with $\left|O_{2 i}\right| / s_{\beta} \simeq 1$ in the $\left|O_{1 i}\right|$ $/ c_{\beta^{-}}\left|O_{2 i}\right| / s_{\beta}$ plane shown in Fig. 5(b), while the rest give $\left|O_{2 i}\right| / s_{\beta}<1$.

Category 2: Another line in Fig. 5(a) corresponds to $\left|\kappa_{V}\right| \simeq 1$ with varying $\left|\kappa_{f}\right|$. This category is related to the vertical line with $\left|O_{1 i}\right| / c_{\beta} \simeq 1$ in Fig. 5(b). From Eq. (59), we know that $\left|O_{1 i}\right| \simeq c_{\beta}$ and $\left|O_{2 i}\right| \simeq s_{\beta}$ could lead to $\left|\kappa_{V}\right| \simeq c_{\beta}^{2}+s_{\beta}^{2}=1$. Nonetheless, the second relation 


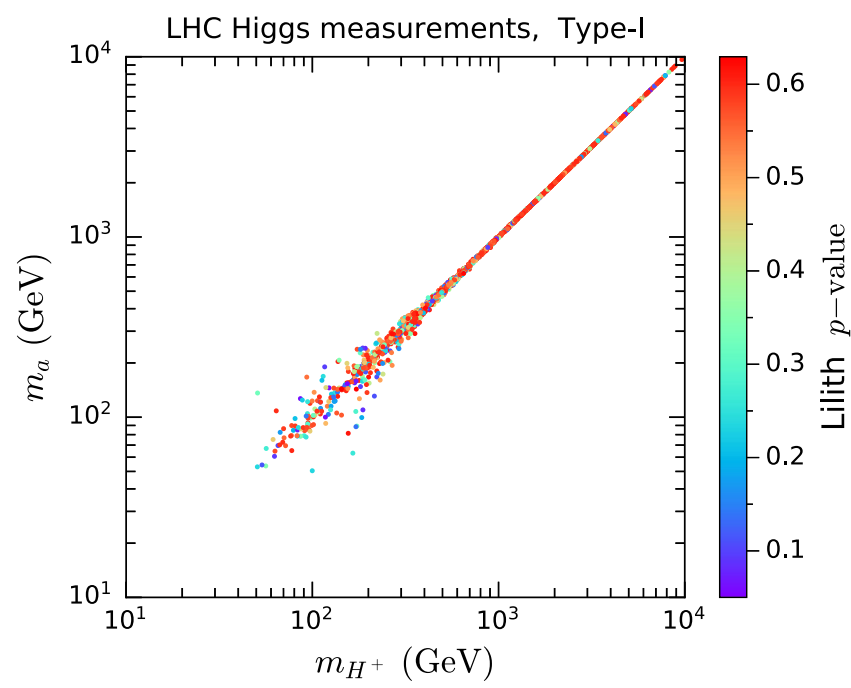

FIG. 4. Lilith $p$-values for the selected parameter points projected in the $m_{H^{+}}-m_{a}$ plane.

$\left|O_{2 i}\right| \simeq s_{\beta}$ is not important to keep $\left|\kappa_{V}\right| \simeq 1$ when $s_{\beta} \ll 1$. Therefore, in the case of $\tan \beta \ll 1,\left|O_{2 i}\right| / s_{\beta}$ could deviate from one, resulting in the vertical line in Fig. 5(b).

There are some scatter points not belonging in the two categories. Most of them correspond to $\tan \beta \sim 1$.

The dominant contributions to $\kappa_{g}$ come from the top and bottom loops, leading to a parametrization of [52]

$$
\kappa_{g}^{2}=1.06 \kappa_{t}^{2}+0.01 \kappa_{b}^{2}-0.07 \kappa_{t} \kappa_{b} .
$$

On the other hand, $\kappa_{\gamma}$ is mainly contributed by the $W$ and top loops, resulting in [52]

$$
\kappa_{\gamma}^{2}=1.59 \kappa_{W}^{2}+0.07 \kappa_{t}^{2}-0.66 \kappa_{W} \kappa_{t} .
$$

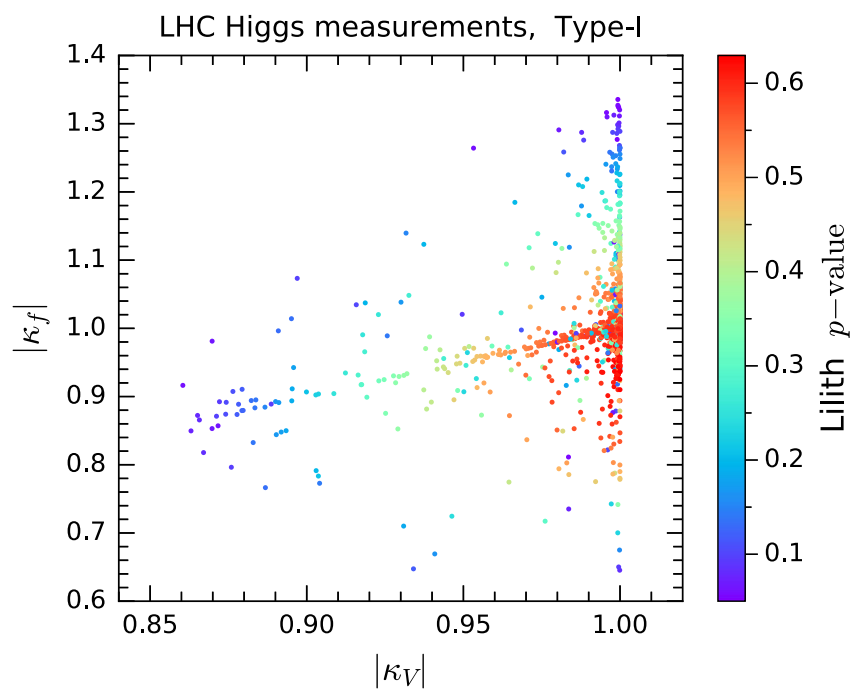

(a) $\left|\kappa_{V}\right|-\left|\kappa_{f}\right|$ plane.
In both cases, the interference between the two contributions gives a term with a negative coefficient. In Fig. 6(a), we project the parameter points in the $\kappa_{g}-\kappa_{\gamma}$ plane, where the points also align along two lines. One line implies a positive correlation between $\kappa_{g}$ and $\kappa_{\gamma}$, corresponding to category 1. This is because the relation $\kappa_{V} \simeq \kappa_{f}$ gives rise to such a positive correlation via Eqs. (63) and (64). On the other hand, when $\left|\kappa_{V}\right| \simeq 1, \kappa_{\gamma}$ is negatively correlated to $\left|\kappa_{f}\right|$ as all selected parameter points satisfy $\kappa_{V} \kappa_{f}>0$. As $\kappa_{g}$ is positively correlated to $\left|\kappa_{f}\right|$, category 2 results in a second line with a negative correlation between $\kappa_{g}$ and $\kappa_{\gamma}$.

$\kappa_{Z \gamma}$ is also dominantly contributed by the $W$ and top loops, given by [52]

$$
\kappa_{Z \gamma}^{2}=1.12 \kappa_{W}^{2}+0.03 \kappa_{t}^{2}-0.15 \kappa_{W} \kappa_{t} .
$$

The correlations of $\kappa_{Z \gamma}$ to $\kappa_{V}$ and to $\kappa_{f}$ are similar to those of $\kappa_{\gamma}$. In addition, $\kappa_{H}$ can be expressed as [52]

$$
\begin{aligned}
\kappa_{H}^{2}= & 0.57 \kappa_{b}^{2}+0.06 \kappa_{\tau}^{2}+0.03 \kappa_{c}^{2}+0.22 \kappa_{W}^{2}+0.03 \kappa_{Z}^{2} \\
& +0.09 \kappa_{g}^{2}+0.0023 \kappa_{\gamma}^{2},
\end{aligned}
$$

where all the coefficients are positive. Thus, $\kappa_{H}$ is positively correlated to both $\left|\kappa_{V}\right|$ and $\left|\kappa_{f}\right|$. The projection in the $\kappa_{Z \gamma}-\kappa_{H}$ plane are shown in Fig. 6(b). Analogous to Fig. 6(a), category 1 leads to a line indicating a positive correlation between $\kappa_{Z \gamma}$ and $\kappa_{H}$ in Fig. 6(b). Besides, parameter points in category 2 roughly align along a second line with a negative correlation.

In Fig. 7(a), we show the projection in the $m_{\chi}-\mathrm{BR}_{\mathrm{inv}}$ plane. When $m_{\chi}>m_{h_{\mathrm{SM}}} / 2$, we have $\mathrm{BR}_{\text {inv }}=0$, because the invisible decay $h_{\mathrm{SM}} \rightarrow \chi \chi$ is kinematically forbidden. When $m_{\chi}<m_{h_{\mathrm{SM}}} / 2$, the invisible branching ratio $\mathrm{BR}_{\mathrm{inv}}$

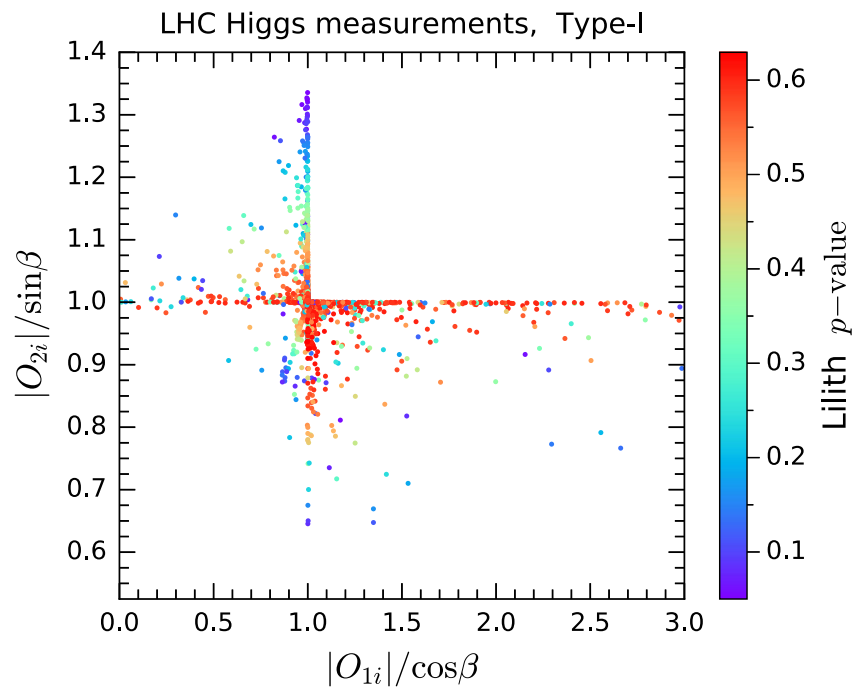

(b) $\left|O_{1 i}\right| / c_{\beta^{-}}\left|O_{2 i}\right| / s_{\beta}$ plane.

FIG. 5. Lilith $p$-values for the selected parameter points projected in the $\left|\kappa_{V}\right|-\left|\kappa_{f}\right|$ (a) and $\left|O_{1 i}\right| / c_{\beta^{-}}\left|O_{2 i}\right| / s_{\beta}$ (b) planes. 


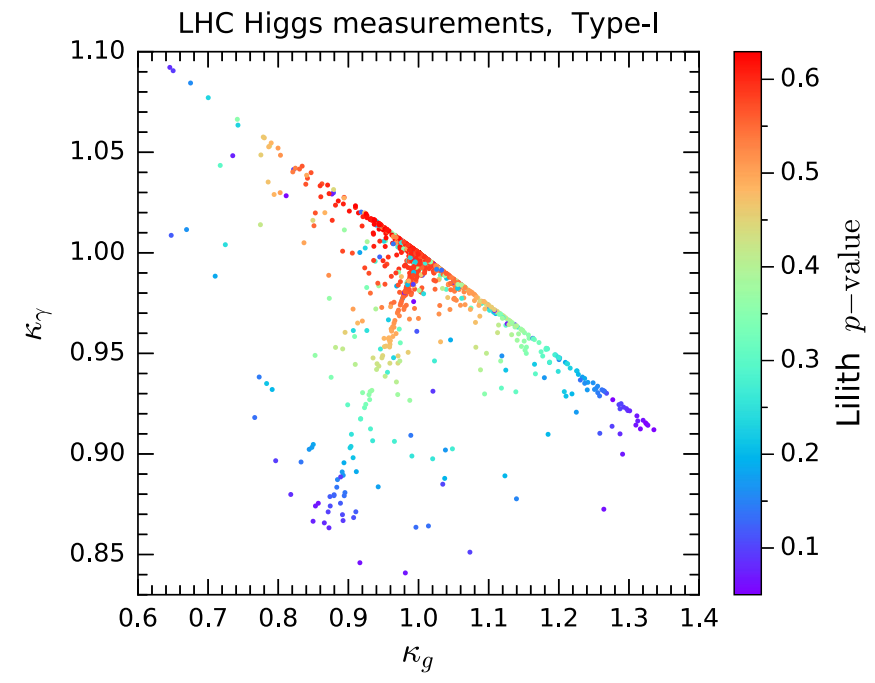

(a) $\kappa_{g}-\kappa_{\gamma}$ plane.

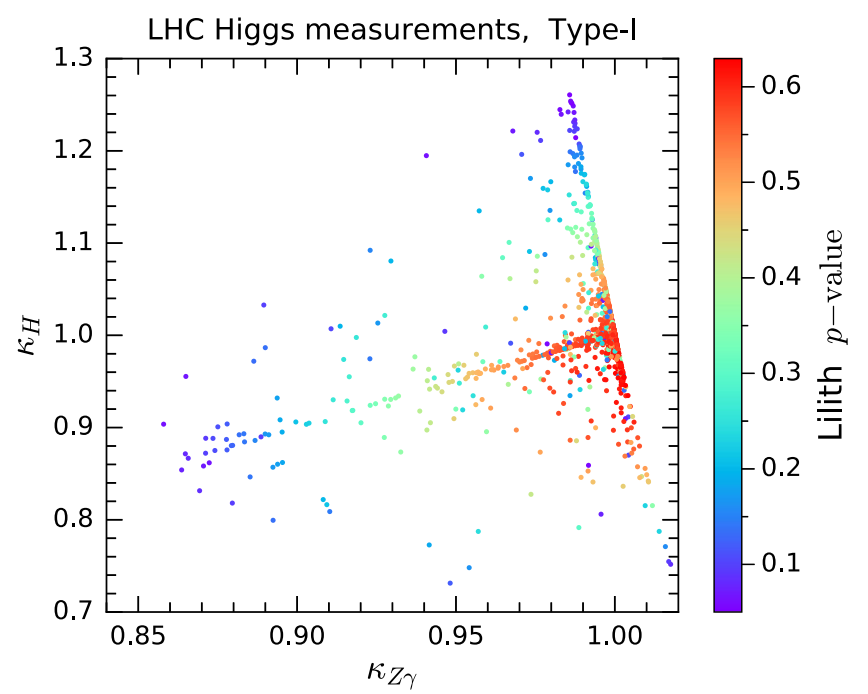

(b) $\kappa_{Z \gamma^{-}} \kappa_{H}$ plane.

FIG. 6. Lilith $p$-values for the selected parameter points projected in the $\kappa_{g}-\kappa_{\gamma}$ (a) and $\kappa_{Z \gamma}-\kappa_{H}$ (b) planes.

could be as large as $\sim 25 \%$ and still consistent with data at 95\% C.L. The projection in the $\Gamma_{h_{\mathrm{SM}}}-\mathrm{BR}_{\text {und }}$ plane is presented in Fig. 7(b). We find that the undetected BSM branching ratio $\mathrm{BR}_{\mathrm{und}}$ can be allowed up to $\sim 27 \%$, while the total width $\Gamma_{h_{\mathrm{SM}}}$ can range from $\sim 2$ to $\sim 7 \mathrm{MeV}$. There is a line implying a positive correlation between $\Gamma_{h_{\mathrm{SM}}}$ and $\mathrm{BR}_{\text {und }}$. This is reasonable, because opening new decay channels enlarges the total width.

In order to investigate the alignment limit, which corresponds to $\tilde{\lambda}_{6}=\tilde{\kappa}_{1}=0$, the selected parameter points are projected in the $\tan \beta-\tilde{\lambda}_{6}$ and $\tan \beta-\tilde{\kappa}_{1}$ planes in Figs. 8(a) and $8(\mathrm{~b})$, respectively. We find that most of the selected points satisfy $\tilde{\kappa}_{1} \simeq 0$, showing no particular dependence on $\tan \beta$. On the other hand, $\tilde{\lambda}_{6}$ is typically close to zero for $\tan \beta \gtrsim 20$ and $\tan \beta \lesssim 0.05$. For $0.05 \lesssim \tan \beta \lesssim 20$, there is no particular favor in the alignment limit.

\section{B. DM relic abundance}

The thermal relic abundance of dark matter is essentially determined by the total velocity-averaged annihilation cross section at the freeze-out epoch, which we denote as $\left\langle\sigma_{\mathrm{ann}} v\right\rangle_{\mathrm{FO}}$. In our model, the DM candidate $\chi$ has the following annihilation channels if kinematically allowed.

(i) Annihilation into a pair of fermions, $\chi \chi \rightarrow f \bar{f}$. This channel is mediated by $s$-channel $C P$-even

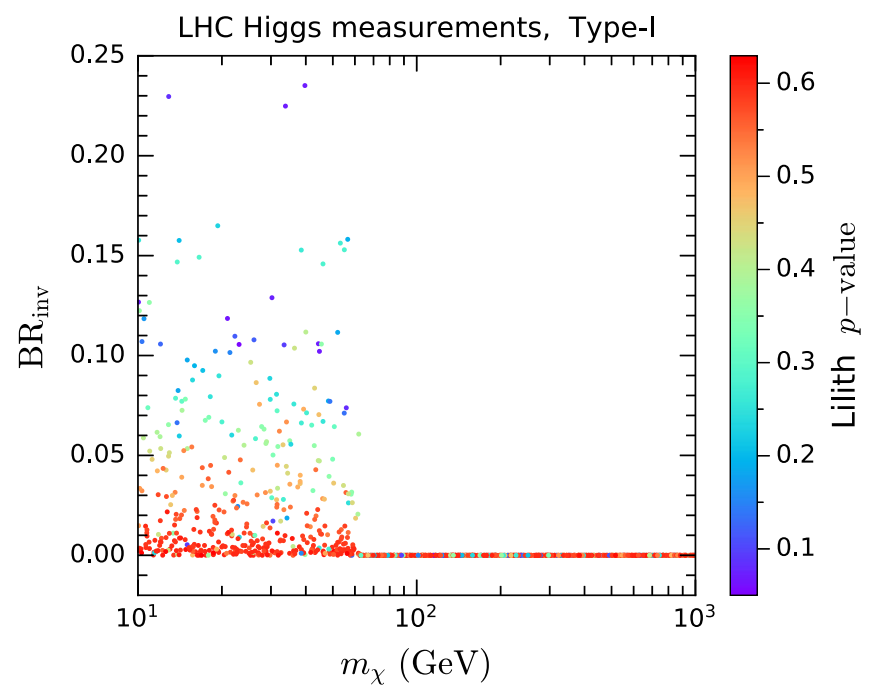

(a) $m_{\chi}-\mathrm{BR}_{\text {inv }}$ plane.

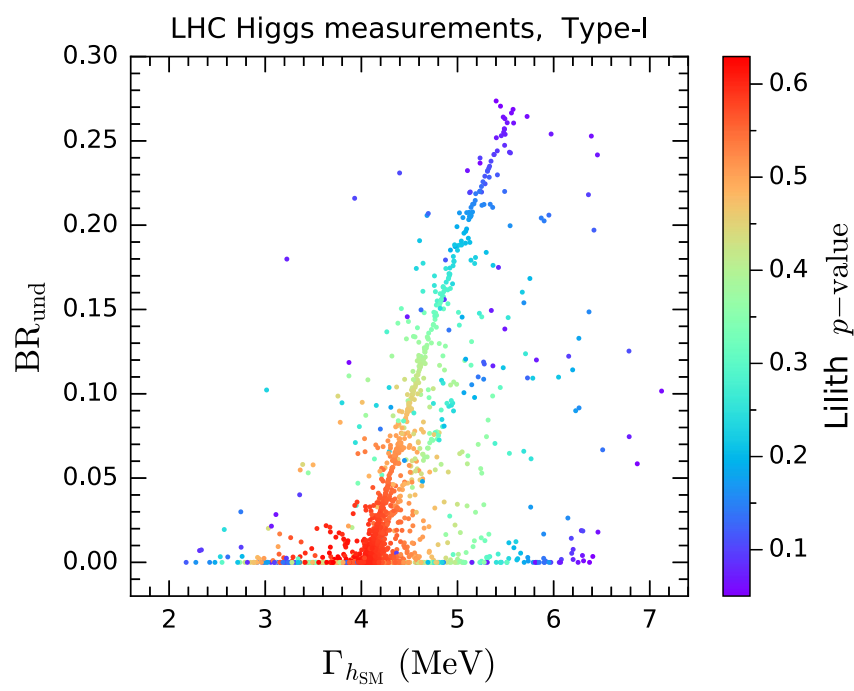

(b) $\Gamma_{h_{\mathrm{SM}}}-\mathrm{BR}_{\text {und }}$ plane.

FIG. 7. Lilith $p$-values for the selected parameter points projected in the $m_{\chi}-\mathrm{BR}_{\mathrm{inv}}$ (a) and $\Gamma_{h_{\mathrm{SM}}}-\mathrm{BR}_{\mathrm{und}}(\mathrm{b})$ planes. 


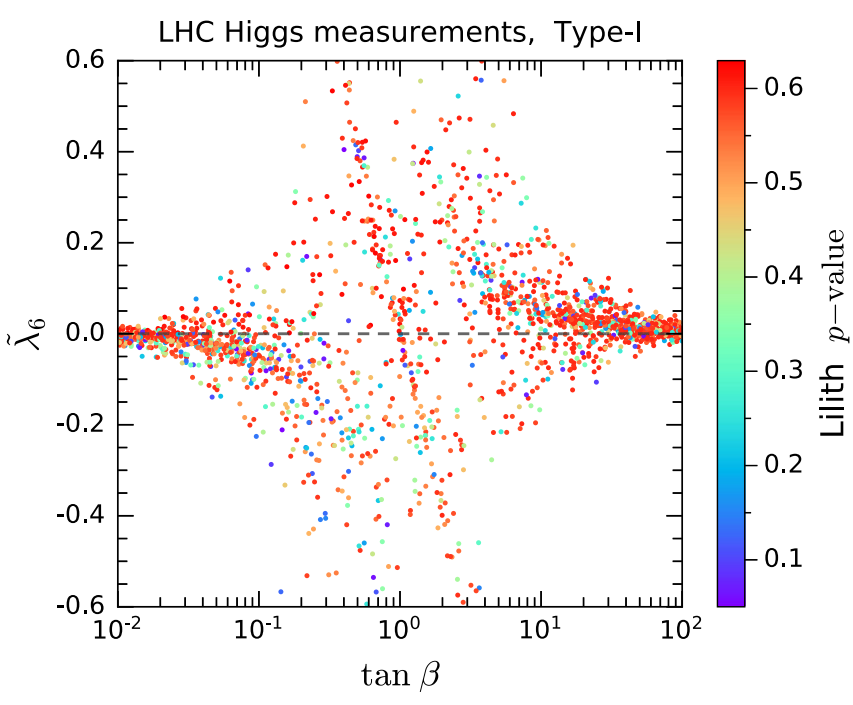

(a) $\tan \beta-\tilde{\lambda}_{6}$ plane.

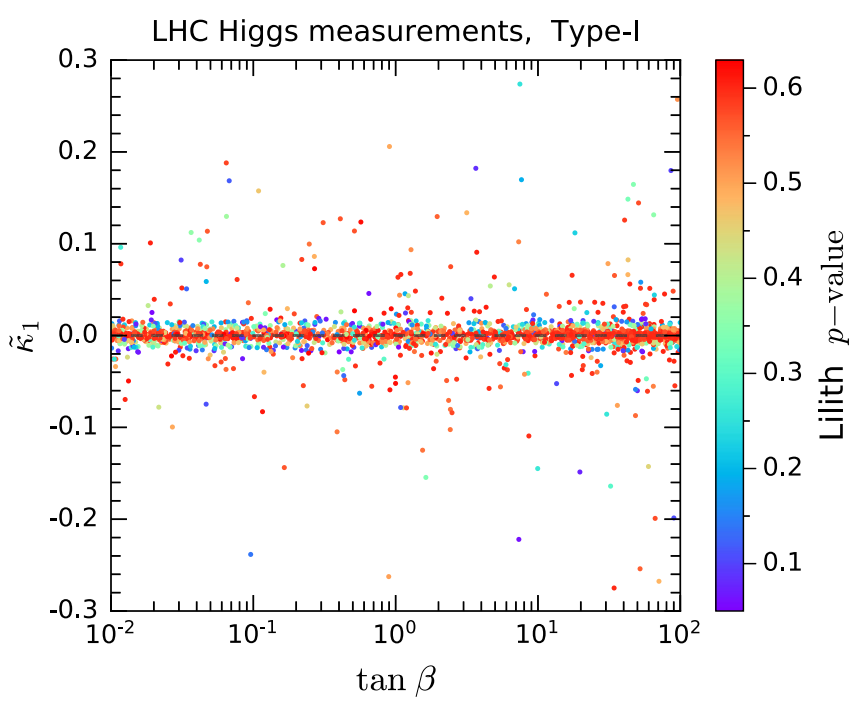

(b) $\tan \beta-\tilde{\kappa}_{1}$ plane.

FIG. 8. Lilith $p$-values for the selected parameter points projected in the $\tan \beta-\tilde{\lambda}_{6}$ (a) and $\tan \beta-\tilde{\kappa}_{1}$ (b) planes. The dashed lines indicate the alignment limit.

Higgs bosons and suppressed by fermion masses. Thus, $t \bar{t}$ and $b \bar{b}$ are the important final states.

(ii) Annihilation into a pair of weak gauge bosons, $\chi \chi \rightarrow W^{+} W^{-}, Z Z$. This channel is also mediated by $s$-channel $C P$-even Higgs bosons.

(iii) Annihilation into a weak gauge boson and a Higgs boson, $\chi \chi \rightarrow W^{ \pm} H^{\mp}, Z a$, mediated by $s$-channel $C P$-even Higgs bosons.

(iv) Annihilation into a pair of $C P$-even Higgs bosons, $\chi \chi \rightarrow h_{i} h_{j}$. This channel can be mediated by $s$-channel $C P$-even Higgs bosons, as well as by $t$ - and $u$-channel $\chi$. Additionally, there are contributions from quartic scalar couplings.

(v) Annihilation into a pair of $C P$-odd or charged Higgs bosons, $\chi \chi \rightarrow a a, H^{+} H^{-}$. This channel is contributed by the mediation of $s$-channel $C P$-even Higgs bosons and quartic scalar couplings.

Some numerical tools are adopted to calculate the relic abundance. We implement the model with FeynRules 2.3.34 [85], and import the generated model files to a Monte Carlo generator MadGraph5_aMC@NLO 2.6.5 [86]. Then we utilize a MadGraph plugin MadDM 3 [87] to compute the relic abundance $\Omega_{\chi} h^{2}$ for each parameter point.

The relic abundance predicted by the selected parameter points is shown in Fig. 9, where the color bar denotes the freeze-out annihilation cross section $\left\langle\sigma_{\mathrm{ann}} v\right\rangle_{\mathrm{FO}}$. We find that the observed value $\Omega h^{2}=0.1186 \pm 0.0020$ given by the Planck experiment [88] corresponds to $\left\langle\sigma_{\mathrm{ann}} v\right\rangle_{\mathrm{FO}} \sim$ $\mathcal{O}\left(10^{-26}\right) \mathrm{cm}^{3} / \mathrm{s}$, which is typical for thermal dark matter. Increase in $m_{\chi}$ typically reduces the annihilation cross section and hence increases the relic abundance. Consequently, if the DM candidate is too heavy, say $m_{\chi} \gtrsim 3 \mathrm{TeV}$, the observed relic abundance could not be achieved.
In Fig. 9, the parameter points predicting $\Omega_{\chi} h^{2}$ over the observed value by $2 \sigma$ are denoted with crosses. These points are considered to be excluded by data, because DM overproduction by the thermal mechanism contradicts standard cosmology. On the other hand, if the predicted thermal relic abundance is too low, there could be some nonthermal production $[89,90]$ occurring after DM freezes out.

\section{Indirect detection}

In this subsection, we discuss constraints from $\gamma$-ray indirect detection experiments. There are couples of

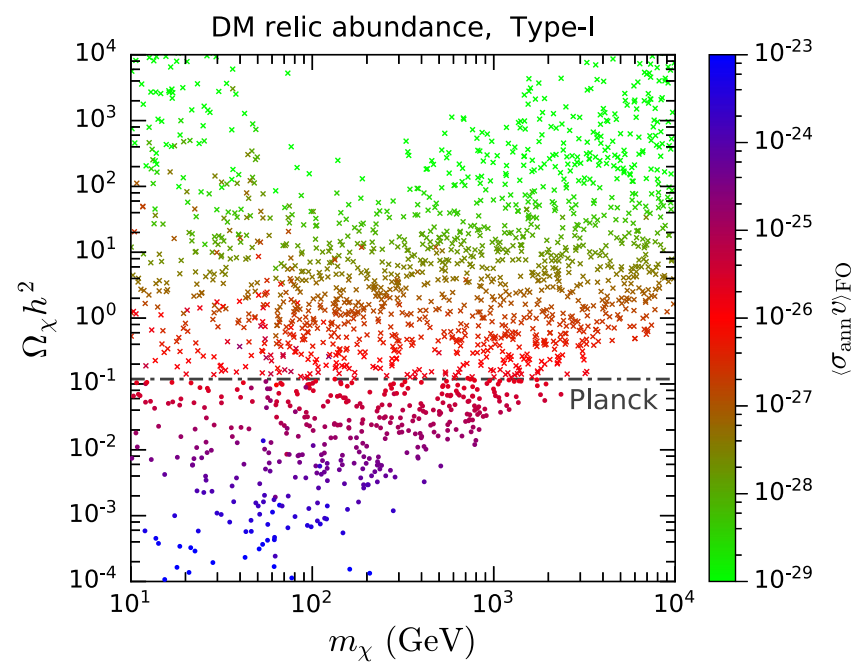

FIG. 9. Selected parameter points projected in the $m_{\chi}-\Omega_{\chi} h^{2}$ plane. The colors indicate the freeze-out annihilation cross section $\left\langle\sigma_{\mathrm{ann}} v\right\rangle_{\mathrm{FO}}$. The dot-dashed line corresponds to the relic abundance measured by Planck [88]. The parameter points denoted with crosses lead to DM overproduction, while those denoted with dots are allowed by data. 
dwarf spheroidal galaxies discovered as satellites of the Milky Way Galaxy. They are considered as the largest substructures of the Galactic dark halo, predicted by the cold DM scenario [91,92]. As known so far, they are the most DM-dominated systems [93]. Moreover, $\gamma$-ray emissions from typical astrophysical sources, such as neutral and ionized gases and recent star formation activity, are expected to be rare in such dwarf galaxies [94-96]. These properties make them perfect targets for searching for $\gamma$-ray emissions from DM annihilation.

The DM velocity dispersion in dwarf galaxies is typically $\sim \mathcal{O}\left(10^{-5}\right)$ [97], which is smaller than DM velocities at the freeze-out epoch by 4 orders of magnitude. Therefore, if the velocity dependence is significant in DM annihilation, the total velocity-averaged cross section in dwarf galaxies $\left\langle\sigma_{\mathrm{ann}} v\right\rangle_{\text {dwarf }}$ could be much different from the freeze-out value $\left\langle\sigma_{\mathrm{ann}} v\right\rangle_{\mathrm{FO}}$.

We further use MadDM to calculate $\left\langle\sigma_{\mathrm{ann}} v\right\rangle_{\text {dwarf }}$ for each parameter point assuming the average DM velocity is $2 \times 10^{-5}$. The ratio of $\left\langle\sigma_{\mathrm{ann}} v\right\rangle_{\text {dwarf }}$ to $\left\langle\sigma_{\mathrm{ann}} v\right\rangle_{\mathrm{FO}}$ is demonstrated in Fig. 10(a), where the parameter points excluded by the Planck relic abundance measurement are not shown. Most of the parameter points give $\left\langle\sigma_{\mathrm{ann}} v\right\rangle_{\mathrm{dwarf}} /$ $\left\langle\sigma_{\mathrm{ann}} v\right\rangle_{\mathrm{FO}} \sim 1$, indicating that $s$-wave annihilation is dominant. Nonetheless, some points give the ratio away from $\mathcal{O}(1)$, indicating significant dependence on velocity. This is typically due to DM annihilation through the resonances of $C P$-even Higgs bosons, since the resonance effect extremely depends on the difference between the resonance location and the velocity-dependent center-of-mass energy $[98,99]$.
The vertical dashed line in Fig. 10(a) indicates the location of $m_{\chi}=m_{h_{\mathrm{SM}}} / 2$, corresponding to the resonance of the SM-like Higgs boson. We can see that the ratio around this line could range from $\sim 10^{-4}$ to $\sim 30$. On the other hand, locations of the other resonances are not fixed, but their effects are also important.

Figure 10(b) shows the projection of the parameter points in the $m_{\chi}-\left\langle\sigma_{\mathrm{ann}} v\right\rangle_{\text {dwarf }}$ plane, as well as the 95\% C.L. upper limits on $\left\langle\sigma_{\mathrm{ann}} v\right\rangle_{\text {dwarf }}$ given by an analysis of Fermi-LAT and MAGIC $\gamma$-ray observations [100]. The analysis combined 6-yr observations of 15 dwarf galaxies from the Fermi-LAT satellite experiment and 158-hr observations of a single dwarf galaxy Segue 1 from the MAGIC Cherenkov telescopes, assuming that the DM distributions in the dwarf galaxies follow the NavarroFrenk-White profile [101]. The limits were obtained assuming that DM solely annihilates into $b \bar{b}$. However, there are various DM annihilation channels in our model. Fortunately, the $\gamma$-ray spectra yielded from these channels should be similar to the spectrum from the $b \bar{b}$ channel, because they are contributed by similar processes, such as hadronization, hadron decays, and final state radiation. Therefore, we have a good reason to expect that the $b \bar{b}$ limits are approximately applicable to our case.

Some parameter points shown in Fig. 10(b) predict a thermal relic abundance lower than the Planck observed value. In this case, the interpretation of indirect detection constraints depends on the assumption of DM composition. If we assume the DM candidate $\chi$ in our model makes up all dark matter in the Universe, nonthermal production $[89,90]$ would be needed to realize the

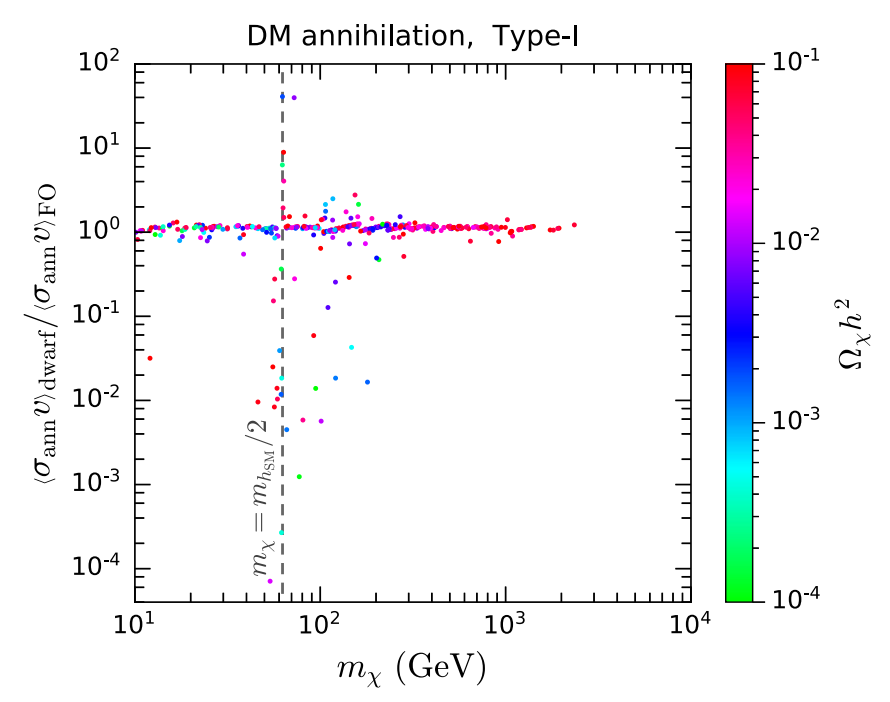

(a) $m_{\chi}-\left\langle\sigma_{\mathrm{ann}} v\right\rangle_{\mathrm{dwarf}} /\left\langle\sigma_{\mathrm{ann}} v\right\rangle_{\mathrm{FO}}$ plane.

FIG. 10. Parameter points with data-allowed relic abundance projected in the $m_{\chi}-\left\langle\sigma_{\text {ann }} v\right\rangle_{\mathrm{dwarf}} /\left\langle\sigma_{\mathrm{ann}} v\right\rangle_{\mathrm{FO}}$ (a) and $m_{\chi}-\left\langle\sigma_{\mathrm{ann}} v\right\rangle_{\mathrm{dwarf}}$ (b) planes. The colors indicate the predicted relic abundance $\Omega_{\chi} h^{2}$. The dashed line in the left panel denotes the location of $m_{\chi}=m_{h_{\mathrm{SM}}} / 2$. The dot-dashed line in the right panel denotes the $95 \%$ C.L. upper limits from $\gamma$-ray observations of dwarf galaxies by Fermi-LAT and MAGIC [100]. 


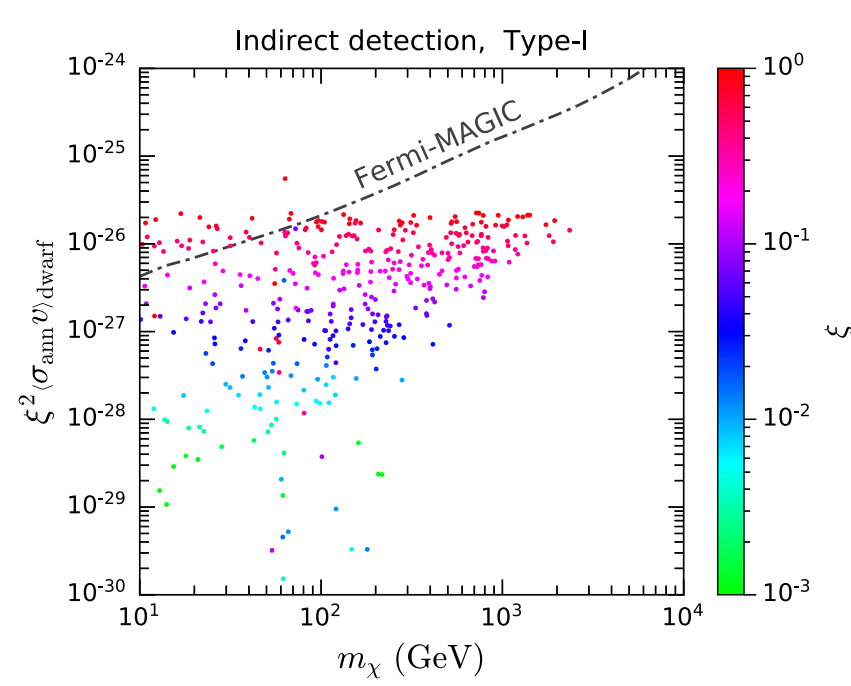

FIG. 11. Parameter points projected in the $m_{\chi}-\xi^{2}\left\langle\sigma_{\text {ann }} v\right\rangle_{\text {dwarf }}$ plane. The colors indicate the ratio of the predicted relic abundance to the observed value, $\xi$. The dot-dashed line denotes the $95 \%$ C.L. upper limits from Fermi-MAGIC $\gamma$-ray observations of dwarf galaxies [100].

observed abundance. Under such an assumption, the Fermi-MAGIC constraint on the parameter space can be directly read off from Fig. 10(b). We can observe that a large fraction of the parameter points with $m_{\chi} \lesssim 1 \mathrm{TeV}$ are ruled out, while the parameter points with $m_{\chi} \gtrsim$ $100 \mathrm{GeV}$ and $\Omega_{\chi} h^{2} \sim 0.1$ are not excluded. Additionally, if $m_{\chi} \simeq m_{h_{\mathrm{SM}}} / 2$, the resonance effect could both yield a data-allowed relic abundance and lead to a small $\left\langle\sigma_{\mathrm{ann}} v\right\rangle_{\mathrm{dwarf}}$ evading the indirect detection constraint.

Another reasonable assumption is that the relic abundance of the DM candidate $\chi$ is exactly predicted by the thermal mechanism, and hence it could only constitute a fraction of all dark matter. The fraction is given by the ratio of the predicted value to the Planck observed value, $\xi \equiv \Omega_{\chi} / \Omega$. Thus, the $\chi \chi$ annihilation cross section in dwarf galaxies should be effectively rescaled to $\xi^{2}\left\langle\sigma_{\mathrm{ann}} v\right\rangle_{\mathrm{dwarf}}$ for comparing with indirect detection constraints. Figure 11 presents the parameter points projected in the $m_{\chi}-\xi^{2}\left\langle\sigma_{\mathrm{ann}} v\right\rangle_{\text {dwarf }}$ plane. Under this assumption, most of the parameter points evade the Fermi-MAGIC constraint.

\section{CONCLUSIONS AND OUTLOOK}

In this paper, we have studied the pNGB DM framework with two $\mathrm{SU}(2)_{\mathrm{L}}$ Higgs doublets $\Phi_{1}$ and $\Phi_{2}$. The DM candidate $\chi$ is the imaginary part of a complex scalar $S$, which is a SM gauge singlet. Most of the scalar potential terms respect a global U(1) symmetry $S \rightarrow e^{i \alpha} S$, except for a soft breaking term giving mass to $\chi$. As a result, $\chi$ becomes a stable massive pNGB. Mass eigenstates in the scalar sector also include three $C P$-even Higgs boson $h_{i}$, a $C P$-odd Higgs boson $a$, and charged Higgs bosons $H^{ \pm}$.
There are four possible types of Yukawa couplings without tree-level FCNCs, just as in the usual twoHiggs-doublet models. DM scattering off nucleons is mediated by the $C P$-even Higgs bosons. Because of the pNGB nature of $\chi$, the scattering amplitude vanishes in the limit of zero momentum transfer for all the four Yukawa coupling types. Although loop corrections lead to a small nonvanishing amplitude, current and near future direct detection experiments are incapable of probing such a DM candidate.

Taking the type-I Yukawa couplings as an example, we have performed a random scan in the 12-dimensional parameter space. The selected parameter points are required to provide a SM-like Higgs boson whose properties are consistent with current LHC Higgs measurements. For $\tan \beta \gg 1$ or $\tan \beta \ll 1$, one of the Higgs doublets acts as the SM-like Higgs doublet, i.e., $\Phi_{2} \simeq \Phi_{h}$ or $\Phi_{1} \simeq \Phi_{h}$, and most of the selected parameter points satisfy $\tilde{\lambda}_{6} \simeq 0$ and $\tilde{\kappa}_{1} \simeq 0$, corresponding to the alignment limit. On the other hand, for $\tan \beta \sim 1$ there is no preference to the alignment limit.

We have also calculated the relic abundance and annihilation cross sections predicted by the selected parameter points. For $m_{\chi} \lesssim 3 \mathrm{TeV}$, it is possible to achieve the observed relic abundance. Because of the resonance effect, the present velocity-averaged annihilation cross section at dwarf galaxies could be rather different from that in the freeze-out epoch. If we assume that $\chi$ makes up all dark matter in the Universe via thermal and nonthermal mechanisms, Fermi-LAT and MAGIC observations of dwarf galaxies have excluded a large fraction of parameter points with $m_{\chi} \lesssim 1 \mathrm{TeV}$. Nonetheless, for $m_{\chi} \simeq m_{h_{\mathrm{SM}}} / 2$ or $100 \mathrm{GeV} \lesssim m_{\chi} \lesssim 3 \mathrm{TeV}$, it is still possible to simultaneously satisfy the constraints from the relic abundance observation and indirect detection. If we assume that $\chi$ only constitutes a fraction of all dark matter when the predicted thermal relic abundance is lower than the observed value, most of the parameter points can evade the Fermi-MAGIC constraint.

Differences among the four types of Yukawa couplings are encoded in the coefficients $\xi_{h_{i}}^{f}$ and $\xi_{a}^{f}$ given by Table I, which lead to different expressions for $\kappa_{b}$ and $\kappa_{\tau}$. Both $\kappa_{b}$ and $\kappa_{\tau}$ in type II are different from those in type I. Thus, we expect the parameter points in type II favored by Higgs measurements should have distinct behaviors from the result present above. On the other hand, $\kappa_{b}$ in the lepton-specific (flipped) Yukawa couplings is identical to that in type I (type II), but $\kappa_{\tau}$ is different. This could cause minor differences in global fits.

Such a pNGB DM model is strongly related to Higgs physics. The proposed future Higgs factories, such as CEPC [102], ILC [103], and FCC-ee [104], would greatly improve the Higgs measurements. We expect that these measurements could significantly restrict the parameter space in our model. Nevertheless, Higgs measurements are 
not able to pin down the DM candidate mass $m_{\chi}$, which is solely determined by the soft breaking term that does not affect the rest of the scalar masses. Thus, indirect detection experiments in the future are essentially important for exploring this model.

\section{ACKNOWLEDGMENTS}

This work is supported in part by the National Natural Science Foundation of China under Grants No. 11805288, No. 11875327 and No. 11905300, the China Postdoctoral Science Foundation under Grant No. 2018M643282, the Natural Science Foundation of Guangdong Province under Grant No. 2016A030313313, the Fundamental Research Funds for the Central Universities, and the Sun Yat-Sen University Science Foundation.

\section{APPENDIX A: SCALAR AND GAUGE TRILINEAR COUPLINGS}

From the scalar potential (4), we derive the scalar trilinear couplings as

$$
\begin{aligned}
\mathcal{L}_{\text {tri }}= & \sum_{i=1}^{3}\left(\frac{1}{2} g_{h_{i} \chi^{2}} h_{i} \chi^{2}+\frac{1}{2} g_{h_{i} a^{2}} h_{i} a^{2}+g_{h_{i} H^{-} H^{+}} h_{i} H^{-} H^{+}\right) \\
& +\sum_{i, j, k}^{3} g_{i j k} h_{i} h_{j} h_{k},
\end{aligned}
$$

where $g_{h_{i} \chi^{2}}$ is already given by Eq. (31), and the other coupling coefficients are given by

$$
\begin{aligned}
g_{h_{i} a^{2}}=- & \left\{\left[s_{\beta}^{2} \lambda_{1}+c_{\beta}^{2}\left(\lambda_{3}+\lambda_{4}-\lambda_{5}\right)\right] v_{1}-2 s_{\beta} c_{\beta} \lambda_{5} v_{2}\right\} O_{1 i} \\
- & \left\{\left[c_{\beta}^{2} \lambda_{2}+s_{\beta}^{2}\left(\lambda_{3}+\lambda_{4}-\lambda_{5}\right)\right] v_{2}-2 s_{\beta} c_{\beta} \lambda_{5} v_{1}\right\} O_{2 i} \\
& -\left(s_{\beta}^{2} \kappa_{1}+c_{\beta}^{2} \kappa_{2}\right) v_{s} O_{3 i}, \\
g_{h_{i} H^{-} H^{+}}= & -\left[\left(s_{\beta}^{2} \lambda_{1}+c_{\beta}^{2} \lambda_{3}\right) v_{1}-s_{\beta} c_{\beta}\left(\lambda_{4}+\lambda_{5}\right) v_{2}\right] O_{1 i} \\
& -\left[\left(c_{\beta}^{2} \lambda_{2}+s_{\beta}^{2} \lambda_{3}\right) v_{2}-s_{\beta} c_{\beta}\left(\lambda_{4}+\lambda_{5}\right) v_{1}\right] O_{2 i} \\
& \quad\left(s_{\beta}^{2} \kappa_{1}+c_{\beta}^{2} \kappa_{2}\right) v_{s} O_{3 i}, \\
g_{i j k}= & -\frac{1}{2}\left(\lambda_{1} v_{1} O_{1 i}+\lambda_{3} v_{2} O_{2 i}+\kappa_{1} v_{s} O_{3 i}\right) O_{1 j} O_{1 k} \\
- & \frac{1}{2}\left(\lambda_{2} v_{2} O_{2 i}+\lambda_{3} v_{1} O_{1 i}+\kappa_{2} v_{s} O_{3 i}\right) O_{2 j} O_{2 k} \\
- & \frac{\lambda_{4}+\lambda_{5}}{2}\left(v_{2} O_{1 i}+v_{1} O_{2 i}\right) O_{1 j} O_{2 k}-\frac{1}{2} \lambda_{S} v_{s} O_{3 i} O_{3 j} O_{3 k} \\
- & \frac{1}{2} \kappa_{1} v_{1} O_{1 i} O_{3 j} O_{3 k}-\frac{1}{2} \kappa_{2} v_{2} O_{2 i} O_{3 j} O_{3 k} \\
- & \frac{1}{2} \kappa_{1} v_{s} O_{3 i} O_{1 j} O_{1 k}-\frac{1}{2} \kappa_{2} v_{s} O_{3 i} O_{2 j} O_{2 k} .
\end{aligned}
$$

By expanding the Lagrangian (22), we obtain the gauge trilinear couplings for the scalars,

$$
\begin{aligned}
\mathcal{L}_{\text {gauge }}= & \sum_{i=1}^{3}\left(g_{h_{i} W W} h_{i} W^{-, \mu} W_{\mu}^{+}+\frac{1}{2} g_{h_{i} Z Z} h_{i} Z^{\mu} Z_{\mu}\right. \\
& \left.+i g_{Z a h_{i}} Z_{\mu} a i \stackrel{\leftrightarrow}{\partial^{\mu}} h_{i}\right)+\sum_{i=1}^{3}\left(g_{W^{ \pm} H^{\mp} h_{i}} W_{\mu}^{+} H^{-} i \stackrel{\leftrightarrow}{\partial^{\mu}} h_{i}\right. \\
& \left.+i \frac{g}{2} W_{\mu}^{+} H^{-} i \stackrel{\leftrightarrow}{\partial^{\mu}} a+\text { H.c. }\right)+e A_{\mu} H^{-} i \stackrel{\leftrightarrow}{\partial^{\mu}} H^{+} \\
& +\frac{g\left(c_{\mathrm{W}}^{2}-s_{\mathrm{W}}^{2}\right)}{2 c_{\mathrm{W}}} Z_{\mu} H^{-} i \stackrel{\leftrightarrow}{\partial^{\mu}} H^{+},
\end{aligned}
$$

where $s_{\mathrm{W}} \equiv \sin \theta_{\mathrm{W}}$. The derivative symbol $\stackrel{\leftrightarrow}{\partial^{\mu}}$ is defined as $F \stackrel{\leftrightarrow}{\partial^{\mu}} G=F \partial^{\mu} G-G \partial^{\mu} F$. The coupling coefficients are given by

$$
\begin{aligned}
g_{h_{i} W W} & =g m_{W}\left(c_{\beta} O_{1 i}+s_{\beta} O_{2 i}\right), \\
g_{h_{i} Z Z} & =\frac{g m_{Z}}{c_{\mathrm{W}}}\left(c_{\beta} O_{1 i}+s_{\beta} O_{2 i}\right), \\
g_{Z a h_{i}} & =\frac{g}{2 c_{\mathrm{W}}}\left(-s_{\beta} O_{1 i}+c_{\beta} O_{2 i}\right), \\
g_{W^{ \pm} H^{\mp} h_{i}} & =\frac{g}{2}\left(-s_{\beta} O_{1 i}+c_{\beta} O_{2 i}\right) .
\end{aligned}
$$

\section{APPENDIX B: BSM DECAY WIDTHS OF THE SM-LIKE HIGGS BOSON}

This Appendix gives the decay widths of the SM-like Higgs boson into two-body BSM final states when they are kinematically allowed. Assuming the SM-like Higgs boson is $h_{\mathrm{SM}}=h_{i}$, its invisible decay width at tree level is

$$
\Gamma\left(h_{i} \rightarrow \chi \chi\right)=\frac{g_{h_{i} \chi^{2}}^{2}}{32 \pi m_{h_{i}}} \sqrt{1-\frac{4 m_{\chi}^{2}}{m_{h_{i}}^{2}}} .
$$

Moreover, its decay widths into $a a$ and $H^{+} H^{-}$are given by

$$
\begin{gathered}
\Gamma\left(h_{i} \rightarrow a a\right)=\frac{g_{h_{i} a^{2}}^{2}}{32 \pi m_{h_{i}}} \sqrt{1-\frac{4 m_{a}^{2}}{m_{h_{i}}^{2}}}, \\
\Gamma\left(h_{i} \rightarrow H^{+} H^{-}\right)=\frac{g_{h_{i} H^{-} H^{+}}^{2}}{16 \pi m_{h_{i}}} \sqrt{1-\frac{4 m_{H^{+}}^{2}}{m_{h_{i}}^{2}} .}
\end{gathered}
$$

Furthermore, the $h_{i} \rightarrow a Z$ decay width can be expressed as

$\Gamma\left(h_{i} \rightarrow a Z\right)=\frac{g_{Z a h_{i}}^{2} m_{h_{i}}^{3}}{16 \pi m_{Z}^{2}} \lambda^{3 / 2}\left(1, m_{a}^{2} / m_{h_{i}}^{2}, m_{Z}^{2} / m_{h_{i}}^{2}\right)$, 
where the $\lambda$ function is defined by

$$
\lambda(x, y, z) \equiv x^{2}+y^{2}+z^{2}-2 x y-2 x z-2 y z .
$$

The decay width of $h_{i} \rightarrow H^{+} W^{-}$is given by

$\Gamma\left(h_{i} \rightarrow H^{+} W^{-}\right)=\frac{g_{W^{ \pm} H^{\mp} h_{i}}^{2} m_{h_{i}}^{3}}{16 \pi m_{W}^{2}} \lambda^{3 / 2}\left(1, m_{H^{+}}^{2} / m_{h_{i}}^{2}, m_{W}^{2} / m_{h_{i}}^{2}\right)$,

which is equal to the decay width of $h_{i} \rightarrow H^{-} W^{+}$.

If $h_{\mathrm{SM}}=h_{2}$ or $h_{3}$, it is possible to decay into $h_{1} h_{1}$ and $h_{2} h_{2}$, whose widths can be commonly expressed as

$$
\Gamma\left(h_{i} \rightarrow h_{j} h_{j}\right)=\frac{\tilde{g}_{i j j}^{2}}{8 \pi m_{h_{i}}} \sqrt{1-\frac{4 m_{h_{j}}^{2}}{m_{h_{i}}^{2}}}
$$

with $\tilde{g}_{i j j}=g_{i j j}+g_{j i j}+g_{j j i}$. If $h_{\mathrm{SM}}=h_{3}$, there is another possible decay channel into $h_{1} h_{2}$. The corresponding width is

$\Gamma\left(h_{3} \rightarrow h_{1} h_{2}\right)=\frac{\tilde{g}_{123}^{2}}{16 \pi m_{h_{3}}} \lambda^{1 / 2}\left(1, m_{h_{1}}^{2} / m_{h_{3}}^{2}, m_{h_{2}}^{2} / m_{h_{3}}^{2}\right)$,

where $\tilde{g}_{123}=g_{123}+g_{231}+g_{312}+g_{213}+g_{132}+g_{321}$.
[1] G. Bertone, D. Hooper, and J. Silk, Particle dark matter: Evidence, candidates and constraints, Phys. Rep. 405, 279 (2005).

[2] J. L. Feng, Dark matter candidates from particle physics and methods of detection, Annu. Rev. Astron. Astrophys. 48, 495 (2010).

[3] B.-L. Young, A survey of dark matter and related topics in cosmology, Front. Phys. 12, 121201 (2017); Erratum, Front. Phys. 12, 121202(E) (2017).

[4] D. S. Akerib et al. (LUX Collaboration), Results from a Search for Dark Matter in the Complete LUX Exposure, Phys. Rev. Lett. 118, 021303 (2017).

[5] X. Cui et al. (PandaX-II Collaboration), Dark Matter Results from 54-Ton-Day Exposure of PandaX-II Experiment, Phys. Rev. Lett. 119, 181302 (2017).

[6] E. Aprile et al. (XENON Collaboration), Dark Matter Search Results from a One Ton-Year Exposure of XENON1T, Phys. Rev. Lett. 121, 111302 (2018).

[7] C. Cheung, L. J. Hall, D. Pinner, and J. T. Ruderman, Prospects and blind spots for neutralino dark matter, J. High Energy Phys. 05 (2013) 100.

[8] S. Banerjee, S. Matsumoto, K. Mukaida, and Y.-L. S. Tsai, WIMP dark matter in a well-tempered regime: A case study on Singlet-Doublets Fermionic WIMP, J. High Energy Phys. 11 (2016) 070.

[9] C. Cai, Z.-H. Yu, and H.-H. Zhang, CEPC precision of electroweak oblique parameters and weakly interacting dark matter: The scalar case, Nucl. Phys. B924, 128 (2017).

[10] T. Han, H. Liu, S. Mukhopadhyay, and X. Wang, Dark matter blind spots at one-loop, J. High Energy Phys. 03 (2019) 080.

[11] W. Altmannshofer, B. Maddock, and S. Profumo, Doubly blind spots in scalar dark matter models, arXiv:1907 .01726.

[12] A. Dedes and D. Karamitros, Doublet-triplet Fermionic dark matter, Phys. Rev. D 89, 115002 (2014).
[13] T. M. P. Tait and Z.-H. Yu, Triplet-quadruplet dark matter, J. High Energy Phys. 03 (2016) 204.

[14] G. Arcadi, C. Gross, O. Lebedev, Y. Mambrini, S. Pokorski, and T. Toma, Multicomponent dark matter from gauge symmetry, J. High Energy Phys. 12 (2016) 081.

[15] C. Cai, Z.-H. Yu, and H.-H. Zhang, CEPC precision of electroweak oblique parameters and weakly interacting dark matter: The Fermionic case, Nucl. Phys. B921, 181 (2017).

[16] Q.-F. Xiang, X.-J. Bi, P.-F. Yin, and Z.-H. Yu, Exploring Fermionic dark matter via Higgs Boson precision measurements at the circular electron positron collider, Phys. Rev. D 97, 055004 (2018).

[17] J.-W. Wang, X.-J. Bi, Q.-F. Xiang, P.-F. Yin, and Z.-H. Yu, Exploring triplet-quadruplet fermionic dark matter at the LHC and future colliders, Phys. Rev. D 97, 035021 (2018).

[18] S. Ipek, D. McKeen, and A. E. Nelson, Renormalizable model for the Galactic Center gamma ray excess from dark matter annihilation, Phys. Rev. D 90, 055021 (2014).

[19] A. Berlin, S. Gori, T. Lin, and L.-T. Wang, Pseudoscalar portal dark matter, Phys. Rev. D 92, 015005 (2015).

[20] J. M. No, Looking through the pseudoscalar portal into dark matter: Novel mono-Higgs and mono-Z signatures at the LHC, Phys. Rev. D 93, 031701 (2016).

[21] D. Goncalves, P. A. N. Machado, and J. M. No, Simplified models for dark matter face their consistent completions, Phys. Rev. D 95, 055027 (2017).

[22] U. Haisch, P. Pani, and G. Polesello, Determining the CP nature of spin- 0 mediators in associated production of dark matter and $t \bar{t}$ pairs, J. High Energy Phys. 02 (2017) 131.

[23] M. Bauer, U. Haisch, and F. Kahlhoefer, Simplified dark matter models with two Higgs doublets: I. Pseudoscalar mediators, J. High Energy Phys. 05 (2017) 138.

[24] P. Tunney, J. M. No, and M. Fairbairn, Probing the pseudoscalar portal to dark matter via $\bar{b} b Z(\rightarrow \ell \ell)+/ E_{T}$ : From the LHC to the Galactic Center excess, Phys. Rev. D 96, 095020 (2017). 
[25] D. Barducci, A. Bharucha, N. Desai, M. Frigerio, B. Fuks, A. Goudelis, S. Kulkarni, G. Polesello, and D. Sengupta, Monojet searches for momentum-dependent dark matter interactions, J. High Energy Phys. 01 (2017) 078.

[26] C. Gross, O. Lebedev, and T. Toma, Cancellation Mechanism for Dark-Matter-Nucleon Interaction, Phys. Rev. Lett. 119, 191801 (2017).

[27] R. Balkin, M. Ruhdorfer, E. Salvioni, and A. Weiler, Dark matter shifts away from direct detection, J. Cosmol. Astropart. Phys. 11 (2018) 050.

[28] K. Huitu, N. Koivunen, O. Lebedev, S. Mondal, and T. Toma, Probing pseudo-Goldstone dark matter at the LHC, Phys. Rev. D 100, 015009 (2019).

[29] T. Alanne, M. Heikinheimo, V. Keus, N. Koivunen, and K. Tuominen, Direct and indirect probes of Goldstone dark matter, Phys. Rev. D 99, 075028 (2019).

[30] K. Kannike and M. Raidal, Phase transitions and gravitational wave tests of pseudo-Goldstone dark matter in the softly broken U(1) scalar singlet model, Phys. Rev. D 99, 115010 (2019).

[31] D. Karamitros, Pseudo Nambu-Goldstone dark matter: Examples of vanishing direct detection cross section, Phys. Rev. D 99, 095036 (2019).

[32] J. M. Cline and T. Toma, Pseudo-Goldstone dark matter confronts cosmic ray and collider anomalies, Phys. Rev. D 100, 035023 (2019).

[33] D. Azevedo, M. Duch, B. Grzadkowski, D. Huang, M. Iglicki, and R. Santos, One-loop contribution to darkmatter-nucleon scattering in the pseudo-scalar dark matter model, J. High Energy Phys. 01 (2019) 138.

[34] K. Ishiwata and T. Toma, Probing pseudo NambuGoldstone boson dark matter at loop level, J. High Energy Phys. 12 (2018) 089.

[35] G. C. Branco, P. M. Ferreira, L. Lavoura, M. N. Rebelo, M. Sher, and J.P. Silva, Theory and phenomenology of two-Higgs-doublet models, Phys. Rep. 516, 1 (2012).

[36] H. E. Haber and G. L. Kane, The search for supersymmetry: Probing physics beyond the Standard Model, Phys. Rep. 117, 75 (1985).

[37] J.E. Kim, Light pseudoscalars, particle physics and cosmology, Phys. Rep. 150, 1 (1987).

[38] N. Turok and J. Zadrozny, Electroweak baryogenesis in the two doublet model, Nucl. Phys. B358, 471 (1991).

[39] P. Ko, Y. Omura, and C. Yu, Higgs and dark matter physics in the type-II two-Higgs-doublet model inspired by $E_{6}$ GUT, J. High Energy Phys. 06 (2015) 034.

[40] N. F. Bell, G. Busoni, and I. W. Sanderson, Self-consistent dark matter simplified models with an s-channel scalar mediator, J. Cosmol. Astropart. Phys. 03 (2017) 015.

[41] C.-F. Chang, X.-G. He, and J. Tandean, Two-Higgsdoublet-portal dark-matter models in light of direct search and LHC data, J. High Energy Phys. 04 (2017) 107.

[42] N. F. Bell, G. Busoni, and I. W. Sanderson, Two Higgs doublet dark matter portal, J. Cosmol. Astropart. Phys. 01 (2018) 015.

[43] A. Dey, J. Lahiri, and B. Mukhopadhyaya, LHC signals of a heavy doublet Higgs as dark matter portal: Cut-based approach and improvement with gradient boosting and neural networks, J. High Energy Phys. 09 (2019) 004.
[44] S. L. Glashow and S. Weinberg, Natural conservation laws for neutral currents, Phys. Rev. D 15, 1958 (1977).

[45] E. A. Paschos, Diagonal neutral currents, Phys. Rev. D 15, 1966 (1977).

[46] D. A. Camargo, M. D. Campos, T. B. de Melo, and F. S. Queiroz, A two Higgs doublet model for dark matter and neutrino masses, Phys. Lett. B 795, 319 (2019).

[47] J. F. Gunion and H. E. Haber, The CP conserving two Higgs doublet model: The approach to the decoupling limit, Phys. Rev. D 67, 075019 (2003).

[48] M. Carena, I. Low, N. R. Shah, and C. E. M. Wagner, Impersonating the Standard Model Higgs Boson: Alignment without decoupling, J. High Energy Phys. 04 (2014) 015.

[49] P. S. B. Dev and A. Pilaftsis, Maximally symmetric two Higgs doublet model with natural Standard Model alignment, J. High Energy Phys. 12 (2014) 024; Erratum, J. High Energy Phys. 11 (2015) 147(E).

[50] H. Georgi and D. V. Nanopoulos, Suppression of flavor changing effects from neutral spinless meson exchange in gauge theories, Phys. Lett. 82B, 95 (1979).

[51] J. F. Donoghue and L. F. Li, Properties of charged Higgs bosons, Phys. Rev. D 19, 945 (1979).

[52] M. Tanabashi et al. (Particle Data Group), Review of particle physics, Phys. Rev. D 98, 030001 (2018).

[53] J. R. Andersen et al. (LHC Higgs Cross Section Working Group), Handbook of LHC Higgs cross sections: 3. Higgs properties, CERN Report No. CERN-2013-004, 2013, 10.5170/CERN-2013-004.

[54] J. Bernon and B. Dumont, Lilith: A tool for constraining new physics from Higgs measurements, Eur. Phys. J. C 75, 440 (2015).

[55] T. Aaltonen et al. (CDF and D0 Collaborations), Higgs boson studies at the Tevatron, Phys. Rev. D 88, 052014 (2013).

[56] G. Aad et al. (ATLAS Collaboration), Search for Invisible Decays of a Higgs Boson Produced in Association with a Z Boson in ATLAS, Phys. Rev. Lett. 112, 201802 (2014).

[57] G. Aad et al. (ATLAS Collaboration), Measurement of Higgs boson production in the diphoton decay channel in pp collisions at center-of-mass energies of 7 and $8 \mathrm{TeV}$ with the ATLAS detector, Phys. Rev. D 90, 112015 (2014).

[58] G. Aad et al. (ATLAS Collaboration), Search for the $b \bar{b}$ decay of the Standard Model Higgs boson in associated $(W / Z) H$ production with the ATLAS detector, J. High Energy Phys. 01 (2015) 069.

[59] G. Aad et al. (ATLAS Collaboration), Search for an invisibly decaying Higgs boson produced via vector boson fusion in $p p$ collisions at $\sqrt{s}=8 \mathrm{TeV}$ using the ATLAS detector at the LHC, CERN Report No. ATLAS-CONF2015-004, 2015.

[60] G. Aad et al. (ATLAS Collaboration), Search for the Standard Model Higgs boson produced in association with top quarks and decaying into $b \bar{b}$ in pp collisions at $\sqrt{s}=$ $8 \mathrm{TeV}$ with the ATLAS detector, Eur. Phys. J. C 75, 349 (2015).

[61] G. Aad et al. (ATLAS Collaboration), Search for the associated production of the Higgs boson with a top quark pair in multilepton final states with the ATLAS detector, Phys. Lett. B 749, 519 (2015). 
[62] G. Aad et al. (ATLAS Collaboration), Study of (W/Z)H production and Higgs boson couplings using $H \rightarrow W W^{*}$ decays with the ATLAS detector, J. High Energy Phys. 08 (2015) 137.

[63] G. Aad et al. (ATLAS Collaboration), Measurements of the Higgs boson production and decay rates and coupling strengths using pp collision data at $\sqrt{s}=7$ and $8 \mathrm{TeV}$ in the ATLAS experiment, Eur. Phys. J. C 76, 6 (2016).

[64] S. Chatrchyan et al. (CMS Collaboration), Search for invisible decays of Higgs bosons in the vector boson fusion and associated $\mathrm{ZH}$ production modes, Eur. Phys. J. C 74, 2980 (2014).

[65] V. Khachatryan et al. (CMS Collaboration), Search for the associated production of the Higgs boson with a top-quark pair, J. High Energy Phys. 09 (2014) 087; Erratum, J. High Energy Phys. 10 (2014) 106(E).

[66] V. Khachatryan et al. (CMS Collaboration), Precise determination of the mass of the Higgs boson and tests of compatibility of its couplings with the standard model predictions using proton collisions at 7 and $8 \mathrm{TeV}$, Eur. Phys. J. C 75, 212 (2015).

[67] V. Khachatryan et al. (CMS Collaboration), Search for a Standard Model Higgs boson produced in association with a top-quark pair and decaying to bottom quarks using a matrix element method, Eur. Phys. J. C 75, 251 (2015).

[68] V. Khachatryan et al. (CMS Collaboration), Search for the standard model Higgs boson produced through vector boson fusion and decaying to $b \bar{b}$, Phys. Rev. D 92, 032008 (2015).

[69] G. Aad et al. (ATLAS Collaboration), Search for new phenomena in the $Z(\rightarrow \ell \ell)+E_{\mathrm{T}}^{\mathrm{miss}}$ final state at $\sqrt{s}=$ $13 \mathrm{TeV}$ with the ATLAS detector, CERN Report No. ATLAS-CONF-2016-056, 2016.

[70] G. Aad et al. (ATLAS Collaboration), Search for the associated production of a Higgs boson and a top quark pair in multilepton final states with the ATLAS detector, CERN Report No. ATLAS-CONF-2016-058, 2016.

[71] G. Aad et al. (ATLAS Collaboration), Search for Higgs boson production via weak boson fusion and decaying to $b \bar{b}$ in association with a high-energy photon in the ATLAS detector, CERN Report No. ATLAS-CONF-2016-063, 2016.

[72] G. Aad et al. (ATLAS Collaboration), Measurement of fiducial, differential and production cross sections in the $H \rightarrow \gamma \gamma$ decay channel with $13.3 \mathrm{fb}^{-1}$ of $13 \mathrm{TeV}$ protonproton collision data with the ATLAS detector, CERN Report No. ATLAS-CONF-2016-067, 2016.

[73] G. Aad et al. (ATLAS Collaboration), Study of the Higgs boson properties and search for high-mass scalar resonances in the $H \rightarrow Z Z^{*} \rightarrow 4 \ell$ decay channel at $\sqrt{s}=$ $13 \mathrm{TeV}$ with the ATLAS detector, CERN Report No. ATLAS-CONF-2016-079, 2016.

[74] G. Aad et al. (ATLAS Collaboration), Search for the Standard Model Higgs boson produced in association with top quarks and decaying into $b \bar{b}$ in $p p$ collisions at $\sqrt{s}=$ $13 \mathrm{TeV}$ with the ATLAS detector, CERN Report No. ATLAS-CONF-2016-080, 2016.

[75] G. Aad et al. (ATLAS Collaboration), Search for the Standard Model Higgs boson produced in association with a vector boson and decaying to a $b \bar{b}$ pair in $p p$ collisions at $13 \mathrm{TeV}$ using the ATLAS detector, CERN Report No. ATLAS-CONF-2016-091, 2016.

[76] G. Aad et al. (ATLAS Collaboration), Measurements of the Higgs boson production cross section via vector boson fusion and associated $W H$ production in the $W W^{*} \rightarrow \ell \nu \ell \nu$ decay mode with the ATLAS detector at $\sqrt{s}=13 \mathrm{TeV}$, CERN Report No. ATLAS-CONF-2016112, 2016.

[77] G. Aad et al. (ATLAS Collaboration), Search for the dimuon decay of the Higgs boson in $p p$ collisions at $\sqrt{s}=$ $13 \mathrm{TeV}$ with the ATLAS detector, CERN Report No. ATLAS-CONF-2017-014, 2017.

[78] V. Khachatryan et al. (CMS Collaboration), First results on Higgs to WW at $\sqrt{s}=13 \mathrm{TeV}$, CERN Report No. CMS-PAS-HIG-15-003, 2016.

[79] V. Khachatryan et al. (CMS Collaboration), VBF H to bb using the 2015 data sample, CERN Report No. CMS-PASHIG-16-003, 2016.

[80] V. Khachatryan et al. (CMS Collaboration), Search for invisible decays of a Higgs boson produced via vector boson fusion at $\sqrt{s}=13 \mathrm{TeV}, \mathrm{CERN}$ Report No. CMSPAS-HIG-16-009, 2016.

[81] V. Khachatryan et al. (CMS Collaboration), Updated measurements of Higgs boson production in the diphoton decay channel at $\sqrt{s}=13 \mathrm{TeV}$ in pp collisions at CMS, CERN Report No. CMS-PAS-HIG-16-020, 2016.

[82] V. Khachatryan et al. (CMS Collaboration), Search for $\mathrm{t} \bar{t} \mathrm{H}$ production in the $\mathrm{H} \rightarrow \mathrm{b} \bar{b}$ decay channel with $2016 \mathrm{pp}$ collision data at $\sqrt{s}=13 \mathrm{TeV}$, CERN Report No. CMSPAS-HIG-16-038, 2016.

[83] V. Khachatryan et al. (CMS Collaboration), Measurements of properties of the Higgs boson decaying into four leptons in pp collisions at sqrts $=13 \mathrm{TeV}, \mathrm{CERN}$ Report No. CMSPAS-HIG-16-041, 2017.

[84] V. Khachatryan et al. (CMS Collaboration), Search for the associated production of a Higgs boson with a top quark pair in final states with a $\tau$ lepton at $\sqrt{s}=13 \mathrm{TeV}, \mathrm{CERN}$ Report No. CMS-PAS-HIG-17-003, 2017.

[85] A. Alloul, N. D. Christensen, C. Degrande, C. Duhr, and B. Fuks, FeynRules 2.0-A complete toolbox for treelevel phenomenology, Comput. Phys. Commun. 185, 2250 (2014).

[86] J. Alwall, R. Frederix, S. Frixione, V. Hirschi, F. Maltoni, O. Mattelaer, H. S. Shao, T. Stelzer, P. Torrielli, and M. Zaro, The automated computation of tree-level and nextto-leading order differential cross sections, and their matching to parton shower simulations, J. High Energy Phys. 07 (2014) 079.

[87] F. Ambrogi, C. Arina, M. Backovic, J. Heisig, F. Maltoni, L. Mantani, O. Mattelaer, and G. Mohlabeng, MadDM v.3.0: A comprehensive tool for dark matter studies, Phys. Dark Universe 24, 100249 (2019).

[88] P. A. R. Ade et al. (Planck Collaboration), Planck 2015 results. XIII. Cosmological parameters, Astron. Astrophys. 594, A13 (2016).

[89] W. B. Lin, D. H. Huang, X. Zhang, and R. H. Brandenberger, Nonthermal Production of WIMPs and the Subgalactic Structure of the Universe, Phys. Rev. Lett. 86, 954 (2001). 
[90] M. Fujii and K. Hamaguchi, Nonthermal dark matter via Affleck-Dine baryogenesis and its detection possibility, Phys. Rev. D 66, 083501 (2002).

[91] V. Springel, J. Wang, M. Vogelsberger, A. Ludlow, A. Jenkins, A. Helmi, J. F. Navarro, C. S. Frenk, and S. D. M. White, The aquarius project: The subhalos of galactic halos, Mon. Not. R. Astron. Soc. 391, 1685 (2008).

[92] J. Diemand, M. Kuhlen, P. Madau, M. Zemp, B. Moore, D. Potter, and J. Stadel, Clumps and streams in the local dark matter distribution, Nature (London) 454, 735 (2008).

[93] L. E. Strigari, Galactic searches for dark matter, Phys. Rep. 531, 1 (2013).

[94] M. Mateo, Dwarf galaxies of the local group, Annu. Rev. Astron. Astrophys. 36, 435 (1998).

[95] J. S. Gallagher, G. J. Madsen, R. J. Reynolds, E. K. Grebel, and T. A. Smecker-Hane, A search for ionized gas in the Draco and Ursa minor dwarf spheroidal galaxies, Astrophys. J. 588, 326 (2003).

[96] J. Grcevich and M. E. Putman, HI in local group dwarf galaxies and stripping by the Galactic halo, Astrophys. J. 696 (2009) 385; Erratum, Astrophys. J. 721, 922(E) (2010).

[97] M. G. Walker, M. Mateo, E. W. Olszewski, J. Penarrubia, N. W. Evans, and G. Gilmore, A universal mass profile for dwarf spheroidal galaxies, Astrophys. J. 704, 1274 (2009); Erratum, Astrophys. J. 710, 886(E) (2010).

[98] P. Gondolo and G. Gelmini, Cosmic abundances of stable particles: Improved analysis, Nucl. Phys. B360, 145 (1991).

[99] K. Griest and D. Seckel, Three exceptions in the calculation of relic abundances, Phys. Rev. D 43, 3191 (1991).

[100] M. L. Ahnen et al. (MAGIC and Fermi-LAT Collaboration), Limits to dark matter annihilation cross-section from a combined analysis of MAGIC and Fermi-LAT observations of dwarf satellite galaxies, J. Cosmol. Astropart. Phys. 02 (2016) 039.

[101] J. F. Navarro, C. S. Frenk, and S. D. M. White, A universal density profile from hierarchical clustering, Astrophys. J. 490, 493 (1997).

[102] M. Dong et al. (CEPC Study Group), CEPC conceptual design report: Volume 2-Physics \& detector, arXiv: 1811.10545.

[103] H. Baer, T. Barklow, K. Fujii, Y. Gao, A. Hoang, S. Kanemura, J. List, H. E. Logan, A. Nomerotski, M. Perelstein et al., The international linear collider technical design report-Volume 2: Physics, arXiv:1306.6352.

[104] A. Abada et al. (FCC Collaboration), FCC-ee: The Lepton collider, Eur. Phys. J. Spec. Top. 228, 261 (2019). 